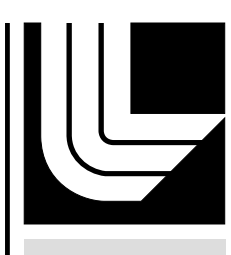

LAW RENCE LIVERMORE N A T IO N A L LABORATORY

\title{
Surface Phenomena and Parameters of Crystal Growth: Simple Basics
}

A. A. Chernov

June 2, 2010

International Summer School on Crystal Growth Dailan, China

August 1, 2010 through August 7, 2010 
This document was prepared as an account of work sponsored by an agency of the United States government. Neither the United States government nor Lawrence Livermore National Security, LLC, nor any of their employees makes any warranty, expressed or implied, or assumes any legal liability or responsibility for the accuracy, completeness, or usefulness of any information, apparatus, product, or process disclosed, or represents that its use would not infringe privately owned rights. Reference herein to any specific commercial product, process, or service by trade name, trademark, manufacturer, or otherwise does not necessarily constitute or imply its endorsement, recommendation, or favoring by the United States government or Lawrence Livermore National Security, LLC. The views and opinions of authors expressed herein do not necessarily state or reflect those of the United States government or Lawrence Livermore National Security, LLC, and shall not be used for advertising or product endorsement purposes. 


\title{
Surface Phenomena and Parameters of Crystal Growth: Simple Basics
}

\author{
A.A.Chernov \\ Lawrence Livermore National Laboratory, 7000 East Avenue, Livermore CA, 94551, USA, chernov2@llnl.gov
}

\begin{abstract}
Basic concepts of crystal growth and their practical use to semi-quantitatively estimate growth processes are explained: surface energy and free energy, driving force of crystallization, atomically rough vs smooth interface structure and the corresponding normal vs layer-by-layer growth modes, application of the activated complex concept to derive kinetic coefficient characterizing crystal growth rate at a given driving force. The Reader is supposed to be familiar with general physics and chemistry. No specific knowledge in crystal growth is required.
\end{abstract}

Keywords. Crystal Growth; Interface; Surface energy; Steps; Kinks; Kinetics; Dislocations; Kinetic coefficient; Roughening transition; Diffuse interface.

PACS: 81.10.Aj;81.10.Dn;81.Be.Be

\section{THE PREFACE}

Physics, chemistry, materials science and other branches of knowledge are becoming more and more sophisticated and are going into subtle chemical and physical details to meet practical engineering challenges. It is hardly possible to memorize all current knowledge, to learn it from the web sites, and to practically make specific materials by just the wide trial and error approach. The only compass in this ocean of knowledge and experience is clear in-depth understanding of general principles.

Crystal growth is not an exception. Its principles have been elaborated since the Gibbs times at the end of the 19-th Century by Wulf, Volmer, Kossel, Stranski, Kaishev, Frank, Cabrera and Burton and many, many other colleagues and are summarized in numerous books and reviews. See, for example, refs [1 - 8] and references therein. In this text, I highlight the basis which may help to feel the roots of some major phenomena, to make simple quantitative estimates of core crystal growth processes, and, via practical application, to deeply feel major general concepts of basic statistical physics. The crystal growth models are still not perfect. Therefore their applicability is also discussed in the text, also to allow better understanding. Of course, selection of subjects is the matter of taste but I tried... The Sections 1,2,3,4 are devoted to the Interface, Driving Force, Layer-by-Layer Growth, and Normal Growth, respectively.

\section{THE INTERFACE}

\subsection{Qualitative Physics.}

Crystal nucleation and growth from vapor, liquid (melt of solution) or other solid are the major phase transition phenomena of the first order. The only phenomenon of that class in addition to crystallization is the vapor-liquid phase transition. All these transitions have one thing in common - an interface 
between the two phases. Existence of the interface means that its free energy, $\gamma$, is positive: $\gamma>0$. Would it be zero or negative, $\gamma<0$, the two phases in contact would intermix down to the molecular scale. Indeed, at $\gamma<0$, the larger the interface area, the lower is total free energy of the system. For example, if a vessel with water and some vapor above it is heated above $373 \mathrm{C}$, the pressure reaches $22 \mathrm{MPa}=22.10^{7} \mathrm{dyn} / \mathrm{cm}^{2}=22.10^{7} \mathrm{erg} / \mathrm{cm}^{2}=220 \mathrm{~atm}$ and the border between liquid and vapor disappears. The vessel is now filled with homogeneous fluid with the density controlled by the initial volumes of the liquid and of the vapor. Under those critical conditions the water-vapor interfacial free energy becomes zero. Similar critical point may be observed in $\mathrm{CO}_{2}$ at its critical temperature $31 \mathrm{C}$ when the pressure reaches 74atm.

A three dimensional (3D) crystal is anisotropic and cannot undergo critical transition to a fluid. However, critical transition may occur on the crystal surface which is a two dimensional (2D) object. In 2D objects, spatial cooperativity of intermolecular interactions is weaker than in three dimensions. Therefore in two dimensional systems thermal vibrations can destroy crystalline order easier than in the 3D case. That transition and the thermal fluctuations from which it originates are of crucial importance for crystal growth. The 2D phase transition on a crystal face may be seen as intermixing between the two following 2D phases. One of these phases is the fully ordered lattice layer (besides some point defects) occupying a part of the crystal face. This layer is limited by the "step"(Fig.1). The surface of this layer is a "smooth" interface. The second 2D phase is on the opposite side from the step. It is nearly empty at the crystal - vapor interface (Fig.1) and is occupied by disordered species at the crystal - melt interface. Attachments of species to and detachment from the step cause the step meandering over the surface. The weaker intermolecular binding energy along the interface and the higher the system temperature the larger is meandering amplitude. When the (energy)/(temperature) ratio (the numbers in Fig.2) is below a critical value, $\sim 0.8$ in Fig.2, the step free energy vanishes, and the meandering spreads over the whole surface layer. That means intermixing between the two 2D phases and is called "roughening" transition. Above the roughening temperature, the whole interface becomes disordered, or "rough" on molecular scale.

At the smooth interface, new species may be attached to the crystal forever only at the "kinks" at the steps (Fig.1) The kink is the end of incomplete molecular row along the step. Therefore the smooth interface grows layer-by-layer, by kink and step propagation. The layer-by-layer growth mode needs step generation. It is therefore is slow and anisotropic and results in polyhedral crystals limited by the smooth facets with some steps separated by atomically flat terraces. Distance between steps is usually 50 to 1000 lattice spacing, depending on growth conditions and material properties, step free energy in the first place. This mode is typical of growth from vapor and solution. On the contrary, the rough interface may acquire new species at about any surface site. Therefore, at the otherwise similar conditions, the growth rate of rough interface is higher and nearly isotropic. This is typical of melt growth resulting in crystal with the rounded growth shape coinciding with the isotherm where $\mathrm{T}=\mathrm{T}_{\mathrm{m}}$, the melting temperature. This shape is determined by temperature distribution. The lower is the free interface energy between a crystal and its vapor, melt or solution, as compared to the thermal energy (both per molecular site), the stronger the fluctuations at the interface, the faster the growth kinetics, the more thorough is statistical "natural selection" of species entering the growing crystal forever. Therefore the interface free energy closely correlates not only with the growth mode, but also with typical defects in the grown crystal. 


\subsection{Interface Geometry and Energetic.}

An ordered interface of the simple cubic lattice is shown in Fig.1. Each cubicle symbolizes an atom, molecule or ion. Incomplete surface lattice layer is terminated by the step. Incomplete row along the step is terminated by the kink. In the crystal bulk the "cubic atom" is bound with 6 first closest neighbors and increasing number of the second, third, etc. neighbors. The work to rupture one bond is designated as $\varepsilon$. The "dangling bond" associated with one missing neighbor is $\varepsilon / 2$. In this simplest approximation, transfer of an atom adsorbed "on the surface" into vacuum requires the work $1 \varepsilon$, the transfer of an atom from the "at the step" position into vacuum needs the work $2 \varepsilon$, an atom "in the kink" needs $3 \varepsilon$, an atom "in the step" needs $4 \varepsilon$, an atom "in the surface" layer needs $5 \varepsilon$, an atom "in the bulk" needs $6 \varepsilon$.

Similar hierarchy of sites exists for other lattices. For instance, in the close packed FCC lattices, the nearest neighbor numbers for the positions on the close packed (111) face, at the close packed step on the (111) face, in the kink, in the step, in the surface and in the bulk are 3,5,6,7,9,12, respectively, not far from the doubled 1,2,3,4,5,6 sequence for simple cubic packing. The reason of this proximity is that both lattices are three dimensional. That proximity justifies why the values of the surface energy, stacking fault energy and some other binding related quantities vary within only $\sim 25 \%$ when estimated making use of either the simple cubic lattice model or a more complex lattice adequate for the material under consideration. That crude approximation might be sometimes useful to avoid tedious analyses of a complex lattice. The detailed analysis, in addition, requires unknown parameters.

The kink position is historically also called "half crystal position". It has special properties because the number of all bonds binding the species in the kink is exactly half of the bonds binding the species in the crystal bulk. In that sense, the kink position is identical for any crystallographic orientation of a step on a face and of the face relative to the crystal lattice - provided that the species are the lattice unit cells as a whole. In other words, the whole cell is considered as the lattice building block, or "building unit". One component materials (like $\mathrm{Si}, \mathrm{Ge}, \mathrm{H}_{2}$, many metals), with the simple cubic (Fig.1), FCC, BCC, HCP, diamond packing, allow each atom to be considered as the building unit though unit cell is several times larger. In these cases, each atom may occupy the kink position. These are well studied Kossel crystals. However, even in the simple $\mathrm{NaCl}$ crystal, there may be two kinds of kinks - the one occupied by $\mathrm{Na}^{+}$the other by $\mathrm{Cl}^{-}$. The same is true for all substances which molecules dissociate while crystal evaporates, dissolves or melts, that is, for overwhelming majority of materials. Majority of molecular crystals, in which identical molecules occupy crystallographically not equivalent positions within unit cell also belong to the non-Kossel class. The non-Kossel crystals became subject of analyses only in this century. Fortunately, however, the macroscopic phenomenological concepts elaborated for the Kossel crystals are of general applicability. 


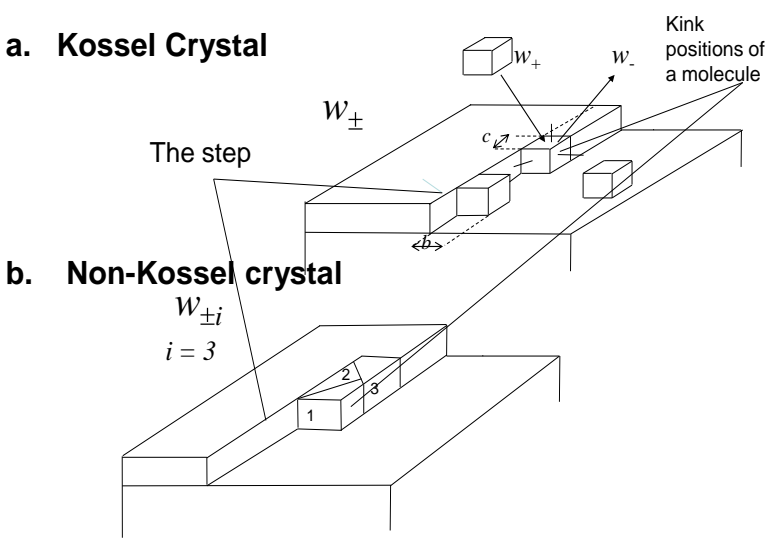

Fig.1
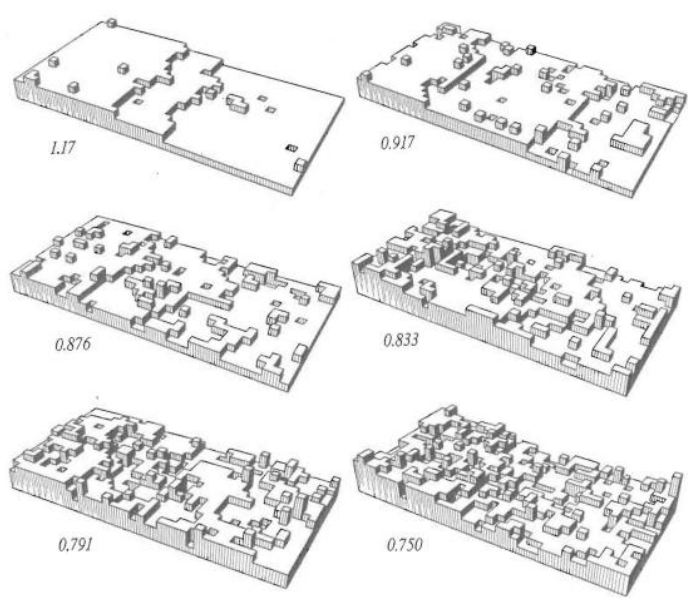

Fig.2

Fig.1. Crystal interface with incomplete layer terminated by the step and the incomplete row terminated by the kink. Molecules/atoms are symbolized by cubes (a) or irregular polygons (b). a. Simple cubic packing with one molecule per unit cell is called Kossel crystal; b. Packing of rectangular unit cells each containing three different molecules/atoms or identical molecules in three not equivalent positions is called non-Kossel crystal.

Fig.2. Monte Carlo computer simulations of the Kossel crystal interface with two steps at different $\varepsilon / 2 \mathrm{kT}$ ratios indicated near the images. As the ratio decreases, the step meandering amplitude rises. Ultimately, the steps disappear though the average interface decline from the close packed (001) orientation determined by the original two steps remains. This is transition from the smooth interface in the upper left to the rough interface in the lower right corner [9].

Each atom on the interface has one or more "dangling valence bonds", i.e. unpaired electrons associated with additional energy. The simplest but rather effective approximation for this energy is $\sim \varepsilon / 2$. Similarly, $\sim \varepsilon / 2$ is the linear step energy per unit site. The free step or surface energy is lower since meandering of the step or the interface rises the entropy. Kink energy is also $\varepsilon / 2$. Attachment of species to or their detachment from the kink does not change number of dangling bonds, thus the interface free energy. Therefore, chemical potential (or free energy, at constant volume) of species in the kink position is the chemical potential (or free energy) of the crystal. For the same reason, minimal work of detachment from the kink is just the transition latent heat,

$\Delta \mathrm{h}=3 \varepsilon$.

This equality allows simply estimate binding energy, $\varepsilon$, and will be considered in more detail below. The $\Delta \mathrm{h}$ is evaporation heat if the species are transferred into vapor or the heat of melting if it is transferred to the melt.

The approach described above and eq.(1) will be subject to further justification. It is good for covalent, metallic and van der Waals bonds but it is far not always valid for ionic crystals, in particularly in solutions, as discussed below. The problems with the ionic crystals stems from the long range nature and strength of electrostatic Coulomb interaction. In some cases, remedy comes from alternation of the positive and the negative ions in the ionic crystals and liquids. For instance, because of that alternation, 
electric field above a surface of ionic crystal in vacuum decreases exponentially. The characteristic distance of that decay is comparable with the lattice spacing. At the interface with ionic liquid screening over Debye radius is added. Therefore operating with multi-ionic neutral species might make the "dangling bond" approach sometimes qualitatively useful.

Crystal growth is driven by difference between chemical potential of crystallizing species in the mother medium $(\mathrm{M})$ - vapor, melt, solution or other solid, $\mu_{\mathrm{M}}$, and chemical potential of a crystal, $\mu_{\mathrm{S}},(\mathrm{S}=\mathrm{solid})$ :

$$
\Delta \mu \equiv \mu_{\mathrm{M}}-\mu_{\mathrm{S}}=\left(\mathrm{u}_{\mathrm{M}}+\mathrm{P} \omega_{\mathrm{M}}-\mathrm{Ts}_{\mathrm{M}}\right)-\left(\mathrm{u}_{\mathrm{S}}+\mathrm{P} \omega_{\mathrm{S}}-\mathrm{Ts}_{\mathrm{S}}\right) .
$$

Here $\mathrm{u}$ is the interaction energy between particles within the phase, $\omega$ is specific volume, $\mathrm{s}$ is the entropy, all per particle, and $\mathrm{P}$ is the pressure. For sufficiently large phases we may ignore surface pressure and stress effects and take the pressures in both phases equal to one another. At phase equilibrium, $\Delta \mu=0$ for all species that enter both the crystal and the medium. First, we will consider only one-component systems built of one type of species, i.e. a crystal in contact with its own melt or vapor. Then the equilibrium condition $\Delta \mu=0$ may be reorganized as follows:

$$
\mathrm{u}_{\mathrm{S}}-\mathrm{u}_{\mathrm{M}}+\mathrm{P}\left(\omega_{\mathrm{S}}-\omega_{\mathrm{M}}\right)=-\mathrm{T}\left(\mathrm{s}_{\mathrm{M}}-\mathrm{s}_{\mathrm{S}}\right)=-\Delta \mathrm{h}
$$

Eq. (3) is equality of the heat function difference on the left to the transition heat $\Delta \mathrm{h}=\mathrm{T}\left(\mathrm{s}_{\mathrm{M}}-\mathrm{s}_{\mathrm{S}}\right)$ on the right. The transitions entropy, $\Delta \mathrm{s}=\mathrm{s}_{\mathrm{M}}-\mathrm{s}_{\mathrm{S}}>0$, since crystal is more ordered and thus posses lower entropy than melt or vapor.

For melting and solidification, the $\Delta \mathrm{s} \sim(1$ to 4$) \mathrm{k}=(1$ to 4$) .1 .38 .10^{-16} \mathrm{erg} / \mathrm{K}$ for elements, $\mathrm{k}$ being the Boltzman constant. For metals, typically $\Delta \mathrm{s}<2 \mathrm{k}$, for complex oxides and organic materials $\Delta \mathrm{s} \sim(2$ to 7)k. That entropy increase naturally comes from increasing of the number of configurations in melt due to the covalent bond anisotropy for elements in the middle of the periodic table, due to the number of different species in complex oxides, and due to orientation variability in melt of these asymmetric oxides species and organic molecules. In crystals and melts, $\omega \sim(1$ to 3$) .10^{-23} \mathrm{~cm}^{3}$, so that at the atmospheric pressure, $\mathrm{P}=10^{6} \mathrm{erg} / \mathrm{cm}^{3}, \mathrm{P} \Delta \omega=\mathrm{P}\left(\omega_{\mathrm{M}}-\omega_{\mathrm{S}}\right)<\sim 0.1$. (1 to 3). $10^{-17} \mathrm{erg} \sim 2.10^{-18} \mathrm{erg}$ where the factor 0.1 stands for typical specific volume change at solidification. For comparison, the fusion enthalpy is much larger: $\Delta \mathrm{h}=8.3 .10^{-13} \mathrm{erg}$ for $\mathrm{Si}$ (melting temperature $\left.\mathrm{T}_{\mathrm{m}}=1678 \mathrm{~K}, \Delta \mathrm{s}=3.6 \mathrm{k}\right), \Delta \mathrm{h}=2.1 .10^{-13} \mathrm{erg}$ for Au $\left(\mathrm{T}_{\mathrm{m}}\right.$ $=1337 \mathrm{~K}, \Delta \mathrm{s}=1.13)$, and $\Delta \mathrm{h}=2 \cdot 3 \cdot 10^{-13} \mathrm{erg}$ for $\mathrm{Fe}\left(\mathrm{T}_{\mathrm{m}}=1811 \mathrm{~K}, \Delta \mathrm{s}=0.91 \mathrm{k}\right)$. Thus, the (pressure) $\mathrm{x}$ (volume) contribution to the heat function difference on the left hand side of eq.(3) is negligible. Consequently, the fusion enthalpy is just difference between interaction energies within the solid and within the liquid rather than the free energies, leaving alone vibration and electronic components.

In case of sublimation, $\mathrm{P} \Delta \omega \sim \mathrm{kT} \sim 1.4 .10^{-13} \mathrm{erg}$ at $\mathrm{T}=1000 \mathrm{~K}$, while $\Delta \mathrm{h}>\sim 10^{-12} \mathrm{erg}$. For example, for $\mathrm{Si}$, $\Delta \mathrm{h} \sim 7.7 .10^{-12} \mathrm{erg}$. Since there is practically no interaction in vapor, the sublimation enthalpy equals the minimal work required to detach an atom or molecule from the kink position justifying eq.(1) for the bond energy in the simple cubic lattice.

Evidently, detachment of species from the crystal bulk or from positions "in the bulk", "in the surface", or "in the step" exceeds $\Delta \mathrm{h}$, since more bonds are to be ruptured. In these cases, vacancies in the bulk, surface layer, or the step are formed and increase the crystal energy. Similarly, detachment of atoms 
adsorbed on a face or a step requires less energy. Only the detachment from the kink may be related to the measured latent heat.

The approach described above is good for the smooth interfaces where the kink and the step are the major element of the interface geometry. However, within the really diffuse interface (Sec.4, Fig.8), all atoms are disordered so that kinks and steps do not exist. This is a case of simple metals in contact with their own melt. Nevertheless, the localized interface approach to this situation like the rough interface in Fig.2 also works and allows for estimates of the interfacial free energy.

The difference between the interaction energies in crystal and in solutions may be far away from the dissolution enthalpy. In this case eq.(1) is invalid. While melting and sublimation always requiring heat supply, dissolution of numerous crystals is accompanied by heating, that is, dissolution generates heat, i.e. is exothermic. For instance, dissolution of $\mathrm{Na}_{2} \mathrm{SO}_{4}$ in water generates $0.28 \mathrm{kcal} / \mathrm{mol}\left(=1.9 .10^{-14} \mathrm{erg}\right.$ per molecule), while dissolution of $\mathrm{Na}_{2} \mathrm{SO}_{4} \cdot 10 \mathrm{H}_{2} \mathrm{O}$ requires $18.7 \mathrm{kcal} / \mathrm{mol}$. Dissolution of $\mathrm{Mn}\left(\mathrm{NO}_{3}\right)_{2}$ generates $\Delta \mathrm{h}=12.7 \mathrm{kcal} / \mathrm{mol}$ while dissolution of $\mathrm{Mn}\left(\mathrm{NO}_{3}\right)_{2} .7 \mathrm{H}_{2} \mathrm{O}$ requires $\Delta \mathrm{h}=-6.1 \mathrm{kcal} / \mathrm{mol}$. Dissolution of $\mathrm{MgI}_{2}, \mathrm{~K}_{2} \mathrm{CO}_{3}, \mathrm{CaBr}_{2}$ generates 50.2, 6.9, 26.3kcal/mol, respectively [3]. The less water enters the structures of $\mathrm{MgSO}_{4}$ and $\mathrm{Na}_{2} \mathrm{CO}_{3}$ the more heat is generated at dissolution of these salts. Would dissolution be just similar to melting or evaporation it should always be accompanied by increase of the system entropy and requires some heat. Heat generation at dissolution suggests that hydration or solvation of species moved from crystal to solution decrease the system energy and generate heat that overwhelms the effect of the entropy increase associated with dissolution. That exothermic effect is naturally the weaker the more water molecules enter the crystal structure so that each ion is hydrated already in the solid and not much is left for hydration at dissolution. Naturally, the crystals dissolving exothermically show retrograde solubility i.e. decrease of the equilibrium concentration with increasing temperature. Fig.3 presents schematic chemical potential versus temperature map for melting (a), regular (b) and retrograde (c) solubility. To capture only the qualitative trend, these simplified plots assume negligible dependence of interaction energy and entropy on temperature. Namely, the energies at $\mathrm{T}=0$ are assumed to be the same as at the phase transition temperature and the Nernst theorem (zero entropy at $\mathrm{T}=0)$ is ignored, since the slope of the $\mu(\mathrm{T})$ lines is constant.

Eqs.(1) - (3) allow to estimate the dangling bond energy from the difference between interaction energies of species in contacting phases. The simplest way from this estimate to the interfacial energy is for a crystal - vapor interface, since there is only negligible interaction in vapor. Then the interface energy is just

$$
\gamma=\varepsilon / 2 a^{2}
$$

where $a^{2}$ is the area per carrier of the dangling bond. However, this estimate presumes that the interface structure is the same as if obtained by cutting the crystal lattice with a plane parallel to the interface and then removing half of the crystal. However, that removal forces the interface species to rearrange, i.e. undergo reconstruction and relaxation.

Reconstruction on the as-cut Si (100) face (Fig. 4a) leads to mutual saturation of two dangling bonds, that is, to pairing of the two unpaired electrons, belonging to neighboring atoms (Fig.4b). That 


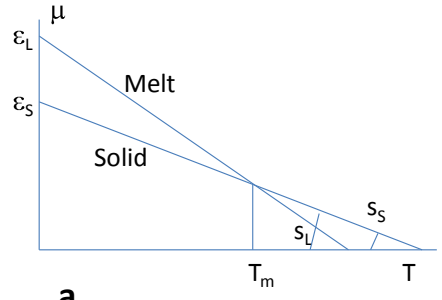

a

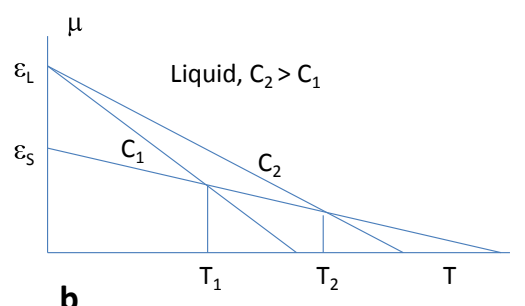

b

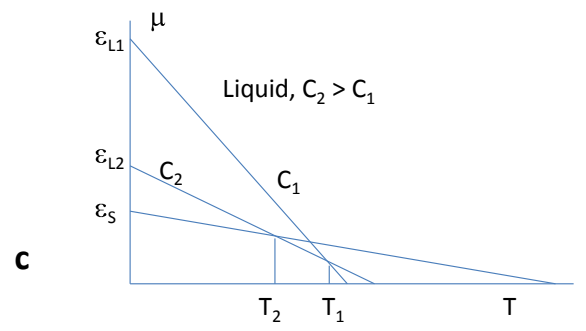

Fig.3. Cross-section between the lines presenting chemical potential $\mu=\mu(\mathrm{T})$ dependence of the crystal-building species, in solid (S) and in liquid (L) provides the equilibrium temperature, T. The crystal - melt system is shown in (a). The system of a crystal and solution containing different concentrations, $C_{1}, C_{2}$, of the crystal species are shown in (b), and (c). The slope $s=-(\partial \mu / \partial T)_{P}$ is the entropy of these species, per particle. The energies and entropies in the phases, $\varepsilon$, $s$, respectively, are assumed to be independent of temperature. $\mathbf{b}$. The crystal - solution system with regular solubility $\mathrm{C}_{\mathrm{e}}(\mathrm{T})$ increasing together with temperature: the solute-solvent interaction $\varepsilon_{L}$ is independent of concentration but the solute entropy in solution naturally decreases with the solute concentration ( $\mu_{L}=k T \ln C \omega+\psi(T)$ ); c. retrograde solubility: entropy is still lower for larger concentration, as in $\mathbf{c}$, but attraction of the solute species to the solvent is stronger than attraction to the neighbors in the crystal.

reconstruction leads to appearance of the $\langle 011\rangle$ dimer rows, surface stress and includes also some distortion of the two deeper (001) layers. This structure is called $\mathrm{Si}(001) 2 \times 1$. The dangling bond saturation, i.e. appearance of the regular (though slightly distorted) covalent electronic bond, resumes the energy of $\sim 2.02 \mathrm{eV} /$ dimer $[10,11]$, i.e. $\sim 10^{3} \mathrm{erg} / \mathrm{cm}^{2}$. For comparison, the free dangling bond energy in the diamond lattice may be taken to be about $1 / 4$ of the sublimation heat, $\Delta \mathrm{h}=4.8 \mathrm{eV} /$ atom $=111 \mathrm{kcal} / \mathrm{mol}=$ $465 \mathrm{~kJ} / \mathrm{mol}$. Related to the unit area per atom $\mathrm{a}^{2} / 2=14.7 .10^{-16} \mathrm{~cm}^{2}$ that gives an estimate of the unreconstructed crystal - vapor interface of $2,600 \mathrm{erg} / \mathrm{cm}^{2}$. This rough estimate for the reconstructed interface gives therefore $\gamma=1,600 \mathrm{erg} / \mathrm{cm}^{2}$. Detailed computations result in the range of $1,490-1,623$ $\mathrm{erg} / \mathrm{cm}^{2}$. Thus reconstruction essentially (by $40 \%$ ) reduces the "cutting-and-separation" work.

Reconstruction also happens on metal - vacuum interface, though with a bit lower correction effect. Different reconstruction on the Ga and As sides of the (111)GaAs interfaces is an example of how the simple dividing of a ruptured bond energy by two to obtain the dangling bond energy is a crude 
approximation.

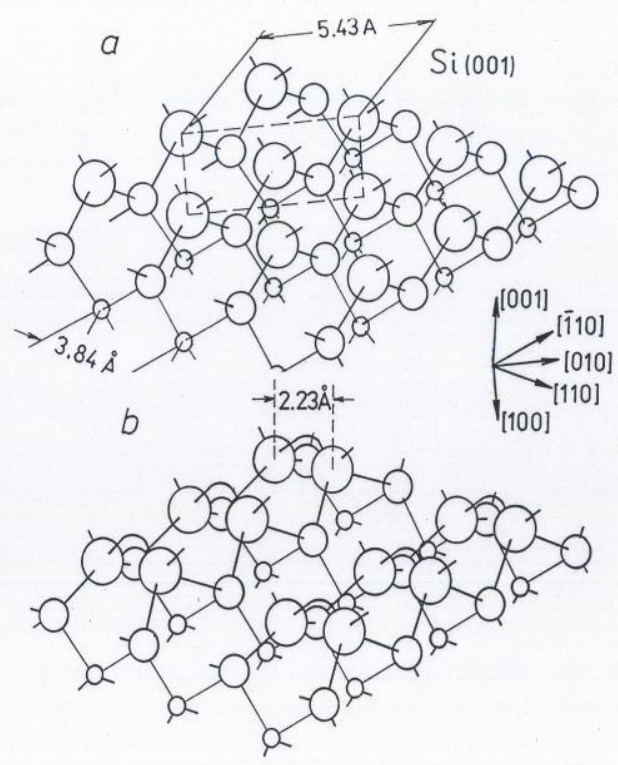

Fig.4. The $\mathrm{Si}(100)$ face: a. just as it is within the ideal crystal lattice; b. after reconstruction, pairing Si atoms along the $\langle 110\rangle$ directions and establishing interatomic distance of $2.23 \mathrm{~A}$ instead of $3.84 \mathrm{~A}$ in the bulk lattice.

Reconstruction is often eliminated by adsorption of species saturating incomplete orbital. For example, the $\mathrm{Si}(111) 7 \mathrm{x} 7$ is transformed to unreconstructed $\mathrm{Si}(111) 1 \mathrm{x} 1$ structure in presence of hydrogen - each $\mathrm{H}$ atom saturates one $\mathrm{sp}^{3}$ dangling bond of one $\mathrm{Si}$ atom on the $\mathrm{Si}$ (111) surface. For the similar saturation reason reconstruction is typically missing on crystal interface with a condensed phase.

Surface relaxation is a change, typically decrease, of the distance between atomic planes closest to the crystal - vacuum interface. It occurs to compensate lack of attraction from the missing half-space.

Relaxation is the strongest (up to $3 \%$ of the lattice spacing) for the "open", least packed faces on metals, like (100),(111)BCC, (110),(311)FCC, [12] but has little effect on the interfacial energy. The relaxation is practically negligible on ionic and weak on covalent crystals.

The dangling bond estimate of the surface energy, eq.(4), is of course crude and does not provide the free energy. Results of experimental measurements of the latter are discussed in the next section.

\subsection{Determining Free Surface Energy.}

The lattice geometry and thermodynamic analysis highlighted above allows an estimate of the bond energy and thus the energy per atomic or molecular site, that is, the interface energy. However, this is 
not yet the free energy of an interface or a step that really matters in crystallization, ripening, ageing and other materials science phenomena. The free interface energy is lower than the energy only by percents if $\varepsilon / 2 \mathrm{kT} \gg>1$. This inequality works at strong binding along the interface and at low temperatures.

However, the free energy may be essentially lower than the energy in the opposite case, when binding within the interface is comparable or lower than the thermal energy. Therefore, more precise data, experimental in the first place, are desirable $[4,13]$.

The free energy of interface (or a step) is the equilibrium property defined as the minimal work required to create a unit area of the interface (or unit length of a step). For instance, theoretically, the interface between phases A and B may be created by cutting both A and B and then connecting A with B and B with $A$. The net work $\varepsilon_{A A}+\varepsilon_{B B}$ is spent to separate the homogeneous phases $A$ and $B$ into two parts each. Then both parts $\mathrm{A}$ are brought into contact with both parts $\mathrm{B}$ creating two interfaces $\mathrm{AB}$. That connection brings back the work $2 \varepsilon_{\mathrm{AB}}$. Surface reconstruction considered in the previous section is an example for the crystal - vacuum interface. The difference divided by the surface area is $2 \gamma_{\mathrm{AB}}$, the doubled interfacial energy between A and B. Alternatively, new AB interface is created when the phase $\mathrm{B}$ is nucleated or grows within the phase A or vice versa. Therefore major data on the free interfacial energies have been experimentally obtained from the rate of crystal nucleation [4]. Grain boundary groove emerging on the crystal - melt interface induces a cusp like groove which shape is also used to evaluate free surface energy. The interface energy also drives Ostwald ripening minimizing total interface in the ensemble of small particles, several $\mu \mathrm{m}$ in diameter or less. Kinetics of that ripening is another source of the free surface energy. Analysis of the equilibrium lens-like shape of a liquid droplet on a crystal is also a working experimental technique. Numerous data on metals are summarized in [13]. Unfortunately, free surface energy averaged over crystallographic orientation of different faces emerged in majority of experiments.

Since the Ostwald ripening was mentioned, it is worth to note that ripening occurs sometimes in saturated solutions in ensembles of crystals that have $10-100 \mu \mathrm{m}$ to $\mathrm{mm}$ size in effective diameter. This is not the Ostwald ripening because in this case the driving force for ripening due to the interface free energy and surface curvature is too small. Instead, growth of the larger crystallites at the expense of the smaller in this case comes from asymmetry between growth and dissolution of bigger and smaller crystals due to the variation of temperature [14].

Melts. Fig.3 [15] is an example of proportionality between the interfacial free energy found in experiments with crystal nucleation in melts and the latent heat of crystallization. The molar surface free energy means the free energy of the surface built of Avogadro number $\left(6.02 .10^{23}\right)$ of sites occupying the area of $\omega^{2 / 3}$ each. The empirical relationship between the melting heat and the solid-melt interface energy is:

$\gamma_{S L} \approx(0.3$ to 0.5$) \Delta h \omega^{-2 / 3}$. 


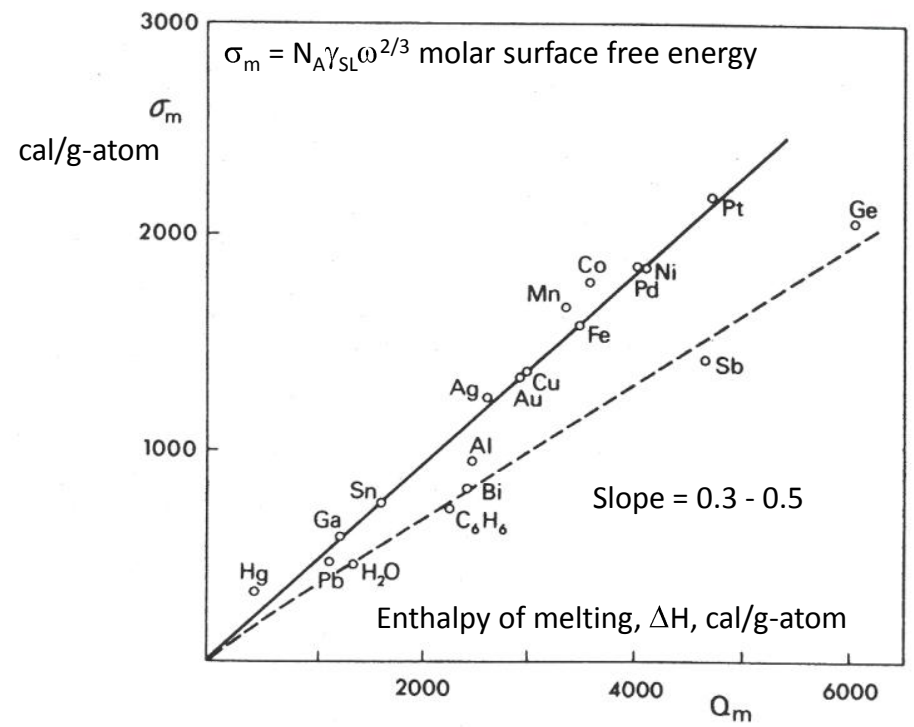

Fig. 1.23. Plot of the gram-atomic interfacial tension $\sigma_{\mathrm{m}}$ vs the molar heat of fusion $Q_{\mathrm{m}}$ for various metals (after Turnbull [84]).

S.Toshev, in Crystal growth- An Intoduction, Ed P.Hartman, NH, 1973 p.1

Fig.5. Proportionality between crystal - melt interfacial free energy and the latent heat of crystallization [15]

Except for the numerical factor ( 0.3 to 0.5 ), this estimate is consistent with the expectation that each atom on the interface occupies an area of about $1 / 6$ of the interface of the "cubic" atom which surface is $\omega^{2 / 3}$ (the empirical coefficient is 1/6.6). The numerical factor, at least partly, takes care of the difference between the just dangling bond energy per a single atomic site, eq.(1), as determined form te difference between interaction energies in the crystal and in the melt, eq.(3), and the really measured free energy of the rough interface. Indeed, the roughening transition means essential increase of the interface entropy. That entropy rise lowers the value of the free energy as compared with the energy per unit site.

Another empirical relationship is related to the typical 5-15\% melting/solidification density change and is useful since the melt - vapor interface free energy, $\gamma_{L V}$ is easier and more reliable to measure than the crystal - vapor interface free energy, $\gamma_{S V}[16]$ :

$\gamma_{S V}=1.13\left(\omega_{L} / \omega_{S}\right)^{2 / 3} \gamma_{L V}=1.13\left(\rho_{S} / \rho_{L}\right)^{2 / 3} \gamma_{L V}$

Here $\rho_{S}, \rho_{L}$ are the solid and melt densities.

Surface melting. Since $\gamma_{S V}>\approx \gamma_{L V}>\gamma_{S L}$, wetting of the "dry" crystal - vapor interface by the melt may occur near the melting temperature, $T_{\mathrm{m}}$. For example, high energy electron diffraction (RHEED) from the $\mathrm{Pb}(110)$ face nearly disappears at $\sim 50 \mathrm{~K}$ below the $\mathrm{Pb}$ melting point, $\mathrm{T}_{\mathrm{m}}=600 \mathrm{~K}$ suggesting that a thin disordered quasiliquid surface layer exists on the crystal - vacuum surface [17]. As the sample temperature approaches the melting point, the thin liquid film is transformed into the bulk melt. The 
"surface melting" occurs not only on the metals surfaces exposed to vacuum but also on $\beta$ methylnaphtalene growing from its vapor and on biphenyl in contact with glass [18]. Experimental and theoretical work on this phenomenon is reviewed in [19]. Thickness of melt film covering the "sweating" crystal surface was measured by Rutherford backscattering to increase from several to tens of atomic/molecular layers and further to became the bulk melt as $T \rightarrow T_{\mathrm{m}}$. The surface melting should be easier if the crystal - vapor interface is smooth while the crystal - melt interface is rough - because the roughening transition is associated with the decrease of the free surface energy. Under that conditions the vapor - solid growth is replaced by the vapor - liquid - solid growth even without foreign component as is the case in the VLS growth mode of nanowires. However, study of the surface melting requires extremely pure material to avoid appearance of liquid eutectics on the crystal interface.

The columns of the Table 1 below [18] list atomic number of the metal, $Z$, its $T_{\mathrm{m}}$, latent heat of fusion per mol, $\Delta \mathrm{H}$, the measured SV, SL, LV surface free energies and the difference

$$
\Delta \equiv \Delta \gamma=\gamma_{S V}-\left(\gamma_{S L}+\gamma_{L V}\right)
$$

between the solid - vapor free energy of the dry interface and the sum of the solid - liquid plus liquid vapor (or another medium) free surface energy of the wetted interface. Thus $\Delta>0$ predicts wetting while the negative sign means that the dry interface has lower free energy than the wetted one. The Table 1 compiles the experimental free interfacial energies. Another tables may be found in $[4,13]$ and other numerous sources.

Solutions. Like with melts, major experimental data on the free surface energy between crystal and solution have been obtained from nucleation experiments. However, in experiments with solutions macroscopic samples have been used rather than the $\sim 100 \mu \mathrm{m}$ droplets in melts. Thus in solutions it is more difficult to eliminate heterogeneous nucleation leading to data scattering and to some undervalued surface free energies. Purity is always a big issue in nucleation measurements. As it was mentioned above, numerous substances dissolve exothermically, i.e. behave opposite to what one may expect from analogy with melting. However, the substances showing "regular" endothermic dissolution show, despite of a big scattering, natural correlation between the free surface energy and solubility. Namely, the crystal - aqueous solution free energy linearly decreases with logarithm of solubility [20]:

$$
\gamma\left(\mathrm{erg} / \mathrm{cm}^{2}\right)=-18.3 \ln C_{e}(\mathrm{~mol} / \mathrm{l})+34.5
$$

or, if calculated per unit surface site of the area $a^{2}$ [35 35. J.Christoffersen, E.Rostrup, M.R.Christoffersen, J.Cryst. Growth, 113,599-605(1991)]:

$$
\gamma a^{2} / k T=-0.272 \ln C_{e}\left(\mathrm{~mol} / \mathrm{m}^{3}\right)+2.82 .
$$


Table 1.

Thermodynamio quantities related to surface melting for a number of common elements. The symbols are defined in the text. $P\left(T=T_{m}\right)$ is the equilibrium vapor pressure at the melting point.

\begin{tabular}{|c|c|c|c|c|c|c|c|c|c|}
\hline$z$ & $\mid \begin{array}{l}T_{m} \\
K\end{array}$ & $\left|\begin{array}{l}\Delta H \\
10^{-21} \\
J / a t\end{array}\right|$ & $\left|\begin{array}{c}\mathrm{sv} \\
\mathrm{mJ} / \mathrm{m}^{2}\end{array}\right|$ & $\begin{array}{c}\mathrm{sl} \\
\mathrm{mJ} / \mathrm{m}^{2}\end{array}$ & $\begin{array}{c}2 v \\
\mathrm{~mJ} / \mathrm{m}^{2}\end{array}$ & $\begin{array}{l}\Delta \\
\mathrm{mJ} / \mathrm{m}^{2}\end{array}$ & $\begin{array}{l}\text { mel- } \\
\text { ting }(+) \\
\text { non- } \\
\text { mel- } \\
\text { ting }(-)\end{array}$ & $\begin{array}{l}T_{\mathrm{m}} \Delta / \mathrm{L} \\
10^{1 \mathrm{a}} \mathrm{K} / \mathrm{m}^{2}\end{array}$ & $\left\{\begin{array}{l}P(T=T) \\
\text { Torr }\end{array}\right.$ \\
\hline 1 & Na 371 & 4.4 & 223 & 31 & 200 & -8 & $(-)$ & -475 & $1 \cdot 10^{-7}$ \\
\hline 121 & $\mathrm{Mg} 923$ & 15.3 & 679 & 115 & 570 & -6 & $(-)$ & -362 & 3 \\
\hline 13 & AI 931.7 & 17.9 & 1032 & 154 & 865 & 13 & $(+)$ & 677 & $3 \cdot 10^{-\infty}$ \\
\hline 14 & Si 1683 & 77.0 & 1038 & 416 & 800 & -178 & $(-)$ & -3891 & $3 \cdot 10^{-4}$ \\
\hline 23 & $\nabla 2003$ & 27.7 & 2280 & 375 & 1900 & 5 & $(+)$ & 362 & $2 \cdot 10^{-2}$ \\
\hline 24 & or 2173 & 24.3 & 2031 & 381 & 1700 & -50 & $(-)$ & -4471 & 3 \\
\hline 251 & Mn 1517 & 24.4 & 1297 & 183 & 1100 & 14 & $(+)$ & 870 & $8 \cdot 10^{-1}$ \\
\hline 26$]$ & $\mathrm{Fe} 1808$ & 26.8 & 2206 & 326 & 1830 & 50 & $(+)$ & 3373 & $2 \cdot 10^{-2}$ \\
\hline 27 & Co 1766 & 26.1 & 2197 & 345 & 1830 & 22 & $(+)$ & 1489 & $2 \cdot 10^{-9}$ \\
\hline 281 & $\mathrm{Ni} 1728$ & 29.3 & 2104 & 356 & 1750 & -2 & $(-)$ & -118 & $3 \cdot 10^{-3}$ \\
\hline 29 & Cu 1356 & 21.7 & 1592 & 263 & 1310 & 19 & $(+)$ & 1187 & $4 \cdot 10^{-4}$ \\
\hline 30 & Zn 692.7 & 47.8 & 895 & 119 & 770 & 6 & $(t)$ & 87 & $1 \cdot 10^{-1}$ \\
\hline 31 & $\mathrm{Ga} 302.9$ & 9.3 & 794 & 58 & 715 & 21 & $(+)$ & 684 & $<1 \cdot 10^{-11}$ \\
\hline 32 & Ge 1232 & 57.6 & 870 & 273 & 640 & -43 & $(-)$ & -920 & $8 \cdot 10^{-7}$ \\
\hline 411 & No 2760 & 40.3 & 2314 & 399 & 1960 & -45 & $(-)$ & -3082 & $8 \cdot 10^{-4}$ \\
\hline 421 & Mo 2883 & 40.3 & 2546 & 490 & 2130 & -74 & $(-)$ & -5294 & $3 \cdot 10^{-2}$ \\
\hline 441 & Ru 2700 & 42.4 & 2591 & 443 & 2250 & -102 & $(-)$ & -6495 & $2 \cdot 10^{-2}$ \\
\hline 45 & $\operatorname{Rh} 2240$ & 36.2 & 2392 & 384 & 1970 - & 38 & $(+)$ & 2351 & $4 \cdot 10^{-3}$ \\
\hline 461 & Pd 1828 & 28.6 & 1808 & 302 & 1480 & 26 & $(+)$ & 1662 & $3 \cdot 10^{-2}$ \\
\hline 47 & $\mathrm{Ag} 1234$ & 20.1 & 1065 & 184 & 910 & -29 & $(-)$ & -1780 & $3 \cdot 10^{-3}$ \\
\hline 48 & Cd 594.1 & 10.1 & 697 & 81 & 590 & 26 & $(t)$ & 1529 & $1 \cdot 10^{-1}$ \\
\hline 49 & In 430 & 6.4 & 638 & 48 & 560 & 30 & $(t)$ & 2016 & $<1 \cdot 10^{-14}$ \\
\hline 50 & Sn 505.1 & 11.8 & 654 & 66 & 570 & 18 & $(+)$ & 770 & $<1 \cdot 10^{-11}$ \\
\hline 73 & $\mathrm{Ta} 3250$ & 52.1 & 2595 & 477 & 2180 & -62 & $(-)$ & -3868 & $5 \cdot 10^{-3}$ \\
\hline 74 & W 3650 & 58.5 & 2753 & 590 & 2340 & -178 & $(-)$ & -11106 & $3 \cdot 10^{-2}$ \\
\hline 75 & $\operatorname{Re} 3440$ & 54.9 & 3100 & 591 & 2650 & -141 & $(-)$ & -8835 & $3 \cdot 10^{-2}$ \\
\hline 76 & Os 2970 & 44.5 & 3055 & 566 & 2500 & -12 & $(-)$ & -801 & $2 \cdot 10^{-2}$ \\
\hline 77 & Ir 2727 & 45.9 & 2664 & 466 & 2250 & -52 & $(-)$ & -3089 & $9 \cdot 10^{-3}$ \\
\hline $78:$ & Pt 2042.5 & 536.2 & 2223 & 334 & 1860 & 29 & $(t)$ & 1636 & $2 \cdot 10^{-4}$ \\
\hline 79 & Au 1336 & 21.0 & 1363 & 200 & 1130 & 33 & $(t)$ & 2099 & $1 \cdot 10^{-5}$ \\
\hline 81 & 1576.8 & 7.2 & 547 & 66 & 465 & 16 & $(+)$ & 1282 & $2 \cdot 10^{-8}$ \\
\hline 82 & $\mathrm{~Pb} 600.7$ & 7.9 & 544 & 62 & 460 & 22 & $(+)$ & 1673 & $4 \cdot 10^{-\infty}$ \\
\hline 83 & Bi 544.2 & 18.3 & 501 & 74 & 380 & 47 & $(+)$ & 1398 & $2 \cdot 10^{-10}$ \\
\hline
\end{tabular}


The dependences eq. (8) and (9) are qualitatively clear: the closer the interaction between the species building the crystal to the interaction of these species with the solvent the smaller is the difference between the crystal and solution, the lower the interfacial energy. On the opposite, sparingly soluble salts show higher interfacial energies in these "foreign" solvents.

\section{DRIVING FORCE.}

The growth rate of an interface, of a step, or of a kink typically increases together with the driving force for crystallization. In solution or vapor growth, the latter is the excess of the actual solution concentration or vapor pressure over the equilibrium value. In melt growth, growth is driven by undercooling $\Delta T=T_{m}$ $-T$ below the melting point. The most general expression for the driving force, is the excess, $\Delta \mu$, of chemical potential of the crystallizing species in the mother medium over the potential in the crystal. This general form is especially needed when several chemical components are involved. The way the growth rate behaves when the driving force increases, is determined by density of the srystallizing species in the medium, by mechanisms controlling incorporation of species into the lattice at the crystal interface, by the supply of these species to the interface, by removal of the reaction products, and by removal or supply of the latent heat of crystallization. The latter problems of mass and heat transport are subject to special analyses, last decades by computer modeling [21, 22].

Many melts contain either many types of different species, like high $\mathrm{Z}$ metals, or the species with tendency to make chains and complexes, like P, Sb, S, or large organic molecules. In these melts, crystal growth rate near $T_{\mathrm{m}}$ first increases with the increase of undercooling and then decreases so that the ultimately liquid disorder may be frozen and a glass formed. The glass is formed at large driving force which, nevertheless remains finite at maximal possible undercooling. Specifically, at the glass formation temperature, the entropy ratio $\left(\mathrm{s}_{\text {glass }}-\mathrm{s}_{\text {crystal }}\right) /\left(\mathrm{s}_{\text {liquid melt }}-\mathrm{s}_{\text {crystal }}\right)=0.38$ for 80 typical glass forming systems [23]. The liquid disorder freezes because viscosity of melt becomes high enough to prevent formation of a crystal rather than a glass. The reason is twofold. One is that mobility of the crystallizing species, for instance large organic molecules, becomes low at low temperatures. The second is that the unit cell of a multicomponent non-Kossel crystal to be formed is large and complicated. That complexity requires long time for all the species, their clusters included, to arrive and to be accommodated at the kink positions. In what follows only the crystal growth kinetics at moderate deviation from equilibrium will be considered.

The difference $\Delta \mu$ between chemical potentials of the mother medium and the crystal is given in the simplest one component system by eq.(2). Dimensionless driving force is often defined as $\Delta \mu / \mathrm{kT}$. In melt or vapor,

$$
\Delta \mu=\Delta \mathrm{s} \Delta \mathrm{T}=\Delta \mathrm{h} \Delta \mathrm{T} / \mathrm{T},
$$

where $\Delta \mathrm{h}$ is the corresponding latent heat and $\mathrm{T}$ is the equilibrium temperature. In ideal vapor or solution,

$$
\Delta \mu=\ln \left[\left(\mathrm{P}-\mathrm{P}_{\mathrm{e}}\right) / \mathrm{P}_{\mathrm{e}}\right], \quad \Delta \mu=\ln \left[\left(\mathrm{C}-\mathrm{C}_{\mathrm{e}}\right) / \mathrm{C}_{\mathrm{e}}\right],
$$


where $\mathrm{P}_{\mathrm{e}}$ and $\mathrm{C}_{\mathrm{e}}$ are equilibrium vapor pressure and solubility, respectively. Typical driving force $\Delta \mu / \mathrm{kT}$ in use to achieve practically employed growth rates are: $10^{-10}$ to $10^{-12}$ for quantum crystallization of ${ }^{4} \mathrm{He}$, $10^{-2}-10^{-5}$ for conventional melt growth, 1 - 5 for sublimation, 20 - 40 for chemical vapor deposition (CVD).

The reciprocal supersaturation, $\mathrm{C}_{\mathrm{e}} /\left(\mathrm{C}-\mathrm{C}_{\mathrm{e}}\right)$, or, in general, $1 /[\exp (\Delta \mu / \mathrm{kT})-1]$ (see below) provides an average measure, how many attachments and detachment of species occur at a lattice site before that site is occupied forever in the process of crystal growth. In other words, the reciprocal supersaturation is a measure of how careful is the process on "natural selection" of species. This selection determines concentration of impurities and point defects in the crystal. For instance, at the rough $\mathrm{Si}$ - melt interface, a lattice site is filled forever only after $10^{4}-10^{5}$ attempts. On the other hand, a lattice site on a smooth (111) facet is filled about 100 times less carefully - because the smooth face grows by 100 times faster propagation of steps. These steps are rare on the growing face and must propagate much faster for the face to keep up with the rough portion of the interface. The larger force to drive the steps to move faster is reached since the facet is left behind of the rough interface. Then the facet turns out to be in the region of deeper undercooling which ultimately pushes the steps to grow faster. Due to the higher driving force ans thus fast growth, for example, concentration of Te impurities trapped by the InSb (111) facet may be 10 times larger than the concentration trapped by the rough interface - at the same macroscopic growth rate normal to these interfaces. Conventional solution growth of $\mathrm{KH}_{2} \mathrm{PO}_{4}$ proceeds at the supersaturation of several percent. Therefore the selection includes only 30-50 trials and errors before filing a lattice site (confer eq.(22) below).

Besides the driving force, the growth rate is proportional to density of the crystallizing species in the mother liquor or gas. That is why high quality thin films are grown from vapor or by CVD at rates of several $\AA / \mathrm{s}$ while melt growth allows the rates of $1-100 \mu \mathrm{m} / \mathrm{s}$ to be employed to grow meter - scale perfect single crystals of Si within a day.

As it was highlighted in Sec 1, a smooth crystal face grows layer-by-layer, via propagation of steps, while rough interface allows attachment of species practically at any place, i.e. it propagates along the normal to itself at any point and is called therefore sometimes normal growth mode. The layer-by layer growth will be considered in Sec.3, the normal growth is discussed in Sec.4.

\section{LAYER-BY-LAYER GROWTH.}

New species may attached to the crystal forever only at the kinks along the steps. Therefore a smooth face, below the roughening transition, grows by steps propagation along that face.

On a clean face, the steps are typically one lattice spacing high though, in general, the steps have tendency to bunch and may form macrosteps micrometer scale high. If the step height is $h$ and the average distance between steps is $\lambda$, the measurable rate $V$ of the face displacement normal to itself is

$$
V=\frac{h}{\lambda} \mathrm{v}_{s t}=p \mathrm{v}_{s t}
$$


The steps may be parallel to one another and form a staircase inclined and propagating in the same direction. In this case, typical of low supersaturations $\Delta \mu / \mathrm{kT} \sim 10^{-3}-10^{-1}$, the ratio $\mathrm{h} / \lambda=p$ is the slope of the staircase, $\mathrm{p} \sim 10^{-2}$ to $10^{-3}$.
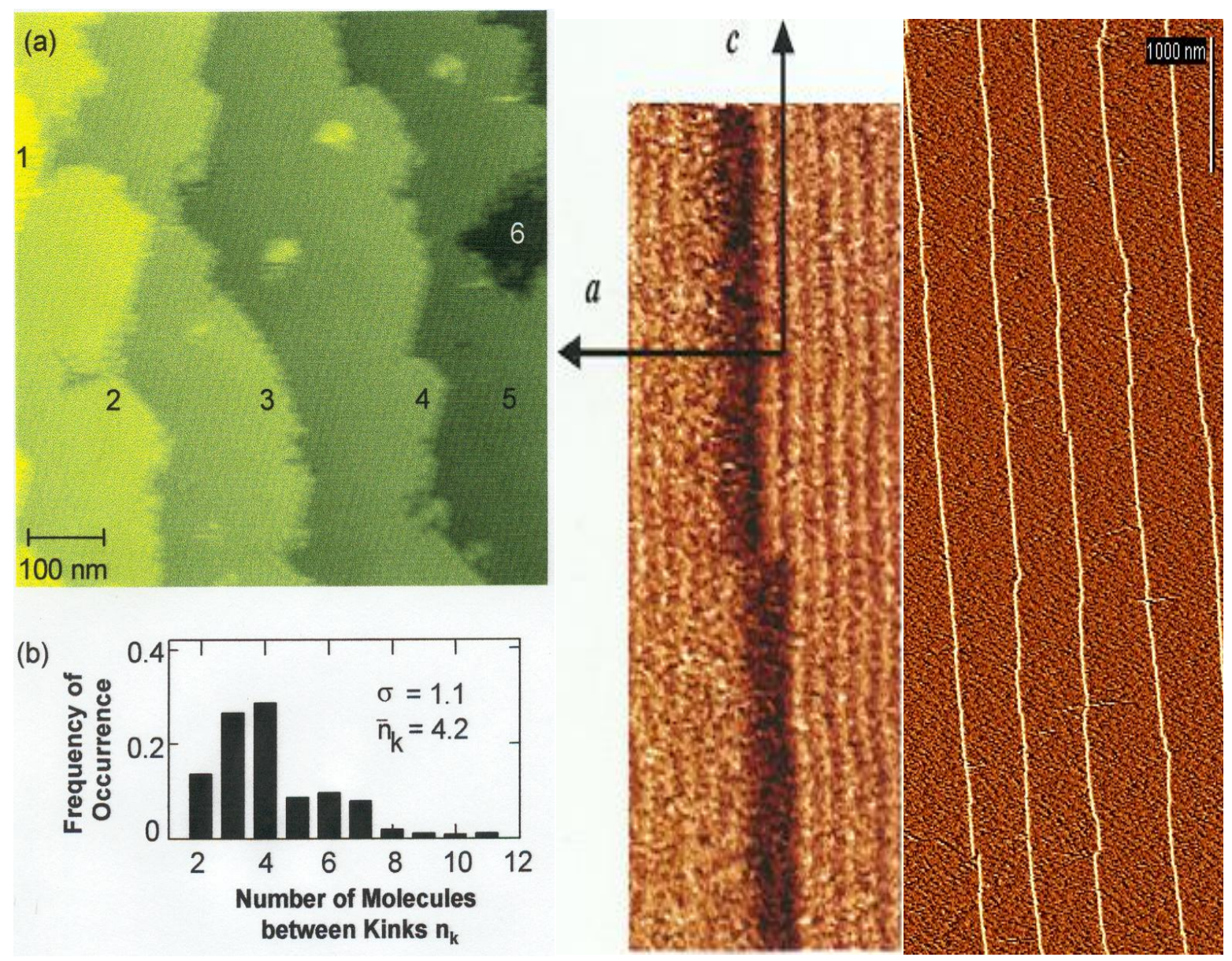

Fig.6.

Fig.7.

Fig. 6. Typical AFM image of the layer-by-layer growth mode on the (111) face of the FCC crystal of ferritin by propagation of steps with high kink density (a). Growth steps are marked as 2 to 6 . Ferritin is the iron storage protein. Its spherical molecules are $13 \mathrm{~nm}$ in diameter and have molecular weight $\mathrm{M}=450,000 \mathrm{Da}$. The steps are separated by smooth terraces. Each step meanders via creation of numerous kinks. Kink density distribution is shown in (b)[24].

Fig.7. Steps with low kink density on orthorhombic (left) and monoclinic (right) polymorph modifications of the protein lysozyme. Each molecule is an ellipsoid with effective diameter of $\sim 2 \mathrm{~nm}, \mathrm{M}=14,300 \mathrm{Da}$. The left image shows single kink which depth is $5.6 \mathrm{~nm}$, equal to the distance between molecular planes seen as vertical stripes. Kink density on the steps seen on the monoclinic crystal on the right is $2.10^{-3} 1 / \mathrm{nm}$, distance between kinks is $490 \mathrm{~nm}$ [courtesy of L.N.Rashkovich]

That staircase geometry is usually generated by screw dislocation or reentrant angle at the foothill of a macrostep. Alternatively, steps may be generated by 2D nucleation on random or preferential points of the smooth terraces between the steps. In that case $h / p$ is an average terrace width.

If $n_{ \pm}(1 / \mathrm{cm})$ is density of both the positive and negative kinks along the step (reciprocal average distance between kinks) and $a$ is the kink depth then the step velocity 
$\mathrm{v}_{s t}=a n_{ \pm} \mathrm{v}_{\mathrm{k}}$

Thus the rate of the layer-by-layer growth of a crystal face is controlled by the rates of step and kink generation and propagation. The kink propagation rate is determined by attachment of new species to the lattice - the core elemental process of crystal growth.

\subsection{Kink Propagation: Activation Complex Concept.}

Vapor, MBE, CVD growth. Atoms of simple materials, like metals, $\mathrm{Si}, \mathrm{Ge}$, attach to the kink site without overcoming a potential barrier because pairing of electrons into complete orbital occurs immediately. Therefore attachment of an atom occurs just as soon as it reaches the kink site. Therefore the step propagation rate is limited just by supply of these atoms and the kink density, as it is clear from eq.(13). The material supply occurs mainly via surface diffusion over the smooth terraces between steps. Indeed, adsorption energy on the terraces may reach $\sim 0.5 \Delta \mathrm{h}$ (for instance, $\sim 2.4 \mathrm{eV}$ for $\mathrm{Si}$ ) so that number density of species in the adsorption layer may exceed that in vapor by orders of magnitude. Potential barrier for surface diffusion on the crystal - vapor interface for atoms or organic molecules is typically 0.2 to 0.5 of the adsorption energy $(1.1 \mathrm{eV}$ for $\mathrm{Si})$. Flux of atoms to a kink from adsorption layer is proportional to the product (adsorption layer density)x(surface diffusivity). For the reasons given above on the adsorption energy and diffusivity barrier, this product strongly exceeds the product (vapor density)x(thermal velocity of atoms)x(kink site area). The latter parameter is the flux of atoms to the kink directly from the vapor. This inequality justifies growth from the adsorption layer. Note that this statement is typically wrong for the growth from melt and solutions where the crystallizing species mostly arrive to the kink from the bulk liquid. Surface diffusion is also essential component of material supply and thus the growth rate of complex semiconductors, like II-VI and III-V compounds from the gas phase. However, for instance, even in the growth of GaAs by molecular beam epitaxy (MBE) the molecules like $\mathrm{As}_{2}, \mathrm{As}_{4}$, or their surface complexes with $\mathrm{Ga}$ are present. Similarly, $\mathrm{Te}_{2}$ complexes participate in CdTe MBE growth. Decomposition of those complexes at kink sites requires formation of an atomic configuration intermediate between that required by surface and that by the kink. Namely, atom(s) not relevant for the specific lattice site at the kink should be detached. For instance, there are no $\mathrm{As}_{2} \mathrm{species}$ in the GaAs lattice. That intermediate "activated complex" has deformed interatomic bonds and thus higher energy than both the initial and final states. Therefore the complex is at the top of the potential barrier for reaction. Numerous different complexes exist on the growing surface and in the gaseous phase during the CVD/MOCVD growth.

Solution and melt . The just described classical Eyring's activated complex concept is applicable also to condensed phases. Let us consider, for example, a kink on a step in a molecular solution. The activated complex may be seen as built of the solvent and solute molecules in one of configurations intermediate between the two following stable configurations. One is a solvated molecule of the crystallizing solute in the solution bulk. The other is the "not activated" solvated kink existing when the next solute molecule is far away from that kink. The activated complex is the configuration corresponding to the saddle point on the surface presenting dependence of the complex energy in the reaction coordinates - interatomic distances or their combinations. On one side from this saddle point is the valley of the solution states of the crystallizing molecule, on the other - the valley of its crystalline 
states. On the border, in the saddle point itself, the energy of the activated complex may be designated as $E$. It may be considered as belonging to either side, that is to either the nearly pure solution ensemble of numerous i-th solution states with the energies $u_{\mathrm{iM}}$ or to the crystal ensemble with the energies $u_{\mathrm{iS}}$. Let us denote the probability for the complex to exist as

$$
p_{i}=A e^{\frac{u_{i}}{k T}}
$$

where we omitted the indices $\mathrm{M}$ and $\mathrm{S}$ at $u_{i}$ since each may be used in the following calculation of the unknown constant A. This constant must satisfy the normalization condition:

$$
\sum_{i} A e^{\frac{u_{i}}{k T}}=1
$$

where the summation takes care of all either solution or crystalline states. The entropy of the crystallizing species (per particle) is:

$$
s=-k<\ln p_{i}>\equiv-k \sum p_{i} \ln p_{i}=-k \sum A e^{\frac{u_{i}}{k T}}\left(\ln A-\frac{u_{i}}{k T}\right)=-k \ln A+\frac{u}{T},
$$

or

$$
T s=-k T \ln A+u, \quad A=e^{\frac{u-T s}{k T}} .
$$

In eq.(16), the brackets $<>$ mean usual averaging over the ensemble of the $i$-th states.

Thus we came to the famous thermodynamic relationship:

$$
p_{i}=e^{\frac{u-T s-u_{i}}{k T}}
$$

For the activated complex, $u_{i}=E$. The difference $u-T s$ is just the free energy per molecule, either in the medium $\mathrm{M}$ (for instance, solution) or in the crystal. In this outline, for simplicity, we assumed the system volume to be constant and thus dropped the term $P \omega_{i}$ in energy. At constant pressure, the normalized probability for the activated complex is expressed via chemical potential, $\mu$, so that the probability to have a crystallizing species sitting in the saddle point and ready to go to both valleys is

$$
p=e^{\frac{\mu-E}{k T}}
$$

Eq. (19) is another classical expression $A=\exp (\mu / k T)$ for normalization constant in the Gibbs distribution at the constant pressure.

A molecule in the activated complex participates in many vibration modes. We designate by $v$ the vibration frequency along the coordinate of crystallization reaction. If electronic transition is crucial, $v \sim$ 
$\mathrm{kT} / \mathrm{h}$, where $\mathrm{h}=6.6 .10^{-27}$ erg.s is the Plank's constant. Then, at $\mathrm{T}=300 \mathrm{~K}$, one may expect $v \sim 6.10^{-12} 1 / \mathrm{s}$. Alternatively, that frequency may be associated with molecular libration or vibration of the molecule as a whole. The uncertainty with the exact mechanism determining the vibration frequency along the reaction coordinate is typical also in diffusion, chemical reactions. Therefore we may only assume $v \sim 10^{13}-10^{12}$ $1 / \mathrm{s}$.

The rate of kink propagation along the step, over the atomic or molecular size $a$ in the lattice, is the difference between the frequencies of attachment, $\mathrm{w}_{+}$, and detachment, $\mathrm{w}_{-}$, both in $1 / \mathrm{s}$, times $a$ :

$$
\mathrm{v}_{\mathrm{k}}=a\left(w_{+}-w_{-}\right)=a v\left(e^{\frac{\mu_{M}-E}{k T}}-e^{\frac{\mu_{s}-E}{k T}}\right)=a v^{\frac{\mu_{s}-E}{k T}}\left(e^{\frac{\mu_{M}-\mu_{S}}{k T}}-1\right)=\beta_{k} \omega\left(C-C_{e}\right), \quad \beta_{k} \equiv a v^{\frac{u_{M}-E}{k T}} .
$$

Here we took into account that at equilibrium the solution concentration $C$ equals its saturation value $C_{e}$ :

$$
\mu_{S}=k T \ln C_{e} \omega+u_{M}
$$

and the kink kinetic coefficient, $\beta_{k}$, was introduced. Together with the kink density, the $\beta_{k}$ controls the measurable kinetic coefficient of a step, eq.(30) below. It is useful to note here the relationships between the attachment and detachment fluxes and the measurable supersaturation $\Delta \mu / \mathrm{kT}$ :

$$
\frac{w_{+}}{w_{-}}=e^{\frac{\Delta \mu}{k T}}=\frac{C-C_{e}}{C_{e}} \text { and the exchange flux } w_{-}=\beta_{k} \omega_{e} / a
$$

The activation barrier $E-u_{\mathrm{M}}$ is the same for both the attachment and detachment of molecules at the kink and, in solutions, may be experimentally estimated from temperature dependence of the step and face growth rate. In the vapor growth and CVD the temperature dependence of the incorporation and the growth rate is overlapped with the temperature dependences of the adsorption layer density and surface diffusivity. In solutions, the activation barrier originates from solvation heat and orientation entropy and should not be confused with the work needed to detach a particle from the kink. The latter includes both the activation barrier and the excess $\Delta \mu$ of chemical potential of the medium over the crystal. The activation barrier is, evidently, the same for growth and decrystallization - dissolution, evaporation, melting, etching.

The driving force, $C-C_{e}$, should, of course, be taken in the immediate vicinity of the kink, over such a small distance that the bulk (or surface) diffusion over that distance is fast as compared with the incorporation rate following from eq.(20). Therefore the local supersaturation at steps and kinks is measured only in the purely kinetic growth mode, when material supply in a (stirred) solution or gas phase is much faster that incorporation processes at the growing interface. The practical signature of the kinetic mode is independence of the growth rate on the material supply rate, for instance, on solution flow in the close vicinity of the growing crystal interface. In other words, the reservoir where solution concentration is known should be at such a distance $\mathrm{L}$ from the growing interface that the typical diffusion rate $\mathrm{D} / \mathrm{L}$ highly exceeds the incorporation rate, i.e. $\mathrm{D} / \mathrm{L} \gg \beta_{\mathrm{st}}$.

Let us now consider growth of ionic crystal from electrolyte solution or generally, of the non-Kossel crystal. The same approach is used for crystallization in which chemical reactions are involved, e.g. in CVD growth. As it was mentioned in Fig1. the unit cell of a non-Kossel crystal includes either 
chemically different species, like anions and cations, or identical molecules (atoms) occupying, however, not equivalent positions. For simplicity, we assume that there are only two kinds of species or two different positions of identical species, A and B, in the unit cell. The crystal cannot grow leaving unfilled sites designated to each of the species. Similarly, it cannot grow leaving behind positions unfilled with identical species. In both cases, the energy increase is too high. Thus the actual "growth unit" is the pair $\mathrm{AB}$ and the driving force replacing $C-C_{e}$ in eq.(20) and others is $C_{A} C_{B}-K$ where the solubility or the reaction equilibrium product $K \equiv e^{\frac{\mu_{A B}-u_{A}-u_{B}}{k T}}$. Here $\mu_{A B}$ is the chemical potential of the AB units in the crystal. The chemical potentials of A and B in solution (or gas) are similar to eq.(21) with the actual concentrations $C_{A}, C_{B}$ replacing $C$. The energies $u_{\mathrm{A}}$ and $u_{\mathrm{B}}$ have the same meaning of the interaction of the solvent with $\mathrm{A}$ and $\mathrm{B}$, respectively. The species taking different positions in the unit cell of a crystal may be identical in solution, as, for instance, protein molecules. In this case, the kink rate

$$
\mathrm{v} \sim C^{2}-C_{e}^{2}=\left(C+C_{e}\right)\left(C-C_{e}\right)
$$

rather than $\sim C-C_{e}$ entering eq.(20) .

In general, kink propagation rate along the step on the non-Kossel crystal is controlled by four frequencies, $w_{+_{A}}, w_{+_{B}}, w_{-A}, w_{-B}[25]$ :

$$
\mathrm{v}_{\mathrm{k}}=a \frac{w_{+A} w_{+B}-w_{-A} w_{-B}}{w_{+A}+w_{+B}+w_{-A}+w_{-B}}
$$

In this equality, $a$ stands for the lattice spacing $\mathrm{AB}$ along the step. The nominator in eq.(24) is $C_{A} C_{B}-K$, as it should be, if the frequencies $\mathrm{w}_{ \pm}$are expressed via chemical potentials and interaction energies are similar to eqs.(18)-(20). The activation barrier entering the kink kinetic coefficient, eq.(20), in this case is $E-u_{A}-u_{B}$. However, eq.(24) is fundamentally different from eq. (20) since it predicts non linear kink rate on the driving force.

Indeed, the basic property of the kink velocity eq.(20), is its linear dependence on the driving force. This linearity follows from statistical independence of attachments and detachments. That independence exists also on the disordered rough interface where even closely located sites behave independently from one another since thermal fluctuations and liquid like rearrangements of the species destroy correlations between the subsequent events. In other words, memory on the past is missing because thermal fluctuations are strong. This is not the case, for instance, in nucleation. Here, average detachment frequency of a particle from a small cluster does depend on the cluster size due to the Gibbs-Thomson law. The cluster size is the result of the previous attachments and detachments. That cooperative memory leads to nucleation barrier, that is, to strongly non-linear dependence of nucleation rate on the driving force, the supersaturation or undercooling. Memory of the previous stage exists also in growth on the non-Kossel crystal surface: the irreversible attachments of A and B must follow one another because, again, violation of this sequence would be too expensive in energy. 
If a non-Kossel crystal grows from stoichiometric solution, $C_{A}=C_{B}$, the kink rate may be reduced to eq.(20), as if the crystal would grow from the $\mathrm{AB}$ molecules, with an effective potential barrier $E-u_{A}-u_{B}$. However, in a non-stoichiometric solution, the kink rate is not linear function of the driving force, $\exp (\Delta \mu / \mathrm{kT})-1$. This non linearity comes from denominator in the eq. (24) where attachment frequencies depend on concentrations of the species A and B. The reason is the predetermined, i.e. strongly correlated $\mathrm{ABAB} \ldots$ sequence of species in the lattice. Physical reason for that non linearity may be understood as follows. If there is a deficit of, say, species B in solution as compared to A, then incorporation of the species B should be the limiting stage of kink propagation. If A is in deficit, the kink rate is determined by incorporation of $\mathrm{A}$. These rates are different even at the same driving force $C_{A} C_{B}-K$. Therefore linearity with the driving force may be expected only when the product $C_{A} C_{B}$ in solution is being changed keeping relative portions of $\mathrm{A}$ and $\mathrm{B}$ constant: $C_{A} / C_{B}=$ const.

\subsection{Step Propagation}

Irreversible attachments of new species to the lattice occur at the kinks on a step. Therefore the step propagation rate is increasing with increasing the kink density unless that density is so high that its further increase does not influence the step rate any more. Most often, the kink density is high, so that the distance between neighboring kinks is only several lattice spacing long. Morphologically, high kink density leads to practical independence of the step rate on its crystallographic orientation within the the face on which the step grow. The isotropic step velocity results in circular shape of a closed step loop. That steps 1-6 are seen in Fig.6. Let us now consider an opposite case of a "singular" step, on which the average distance between kinks is tens of lattice spacing long (Fig.7). Typically, such singular steps have simple crystallographic Miller indices since they are oriented along the closest packed directions in the crystal lattice. If a step is essentially declined from this singular orientation it has much more kinks for just geometrical reason: the crystal lattice is descrete. Therefore, at the same driving force, the declined step will grow much faster than the singular one. What will it be the final shape of a step that form a closed loop? Evidently, the step portions with high kink density grow faster and wedge out leaving on the final steady state growth shape only the slowest orientations. The singularly oriented steps are usually the slowest, so that the steady state loop will take polygonal shape of which edges are these singular orientations. Fig.7 shows a part of that edges. Thus the step morphology is a tool to penetrate into molecular growth processes.

At and away from equilibrium, kinks are produced on a step by random thermal detachments and attachments of species. The kinks may also mutually annihilate either elongating or shrinking the segments of the molecular row they terminate. Rates of the kink birth and annihilation may therefore depend on driving force. In what follows we first consider the most often case when the kink density is high and is controlled by equilibrium thermal fluctuation. The equilibrium kink concentration is determined by its energy. This kink energy is the work required to create a new kink, similar to the work to create a new surface. In a simple Kossel cubic lattice, the kink energy is $\varepsilon / 2$, that is the energy of one "dangling" bond. On a long step of, say, <100> orientation on the (001) face, number of species forming the step edge equals the number of contacts between these species - if the end effects are ignored.

Therefore the kink at each connection (contact) between the neighbors may appear independently from 
the kinks at the other connections - in the sense that appearance of one kink does not require appearance of another and thus does not require additional change in energy. The simplest way to feel this independence is to make a kink as follows. Let is consider a horizontal $\langle 100\rangle$ step limiting a semiinfinite lattice layer below it. To create a new kink, let us cut the layer by the normal to the average step orientation along the connection where the new kink is supposed to be. Then let us shift half of the layer located on, say, the left hand side of from the cut up in the direction normal to the step by one lattice spacing. That shift creates one kink and increases the system energy by $\varepsilon / 2$. All the step configurations on the both the left and right sides of the cut remain intact. That means lack of cooperative interaction along the step. Therefore the kinks are independent from one another and thus must be randomly distributed along the step. The kinks form the 1D gas. Note that a similar operation of creating one single kink on a surface independently of the others is impossible. Indeed, on a square lattice of atoms, the number of contacts between atoms where the kinks may be is twice the number of atoms. That impossibility to create on kink independently of the others demonstrates cooperative interaction on that square, the $2 \mathrm{D}$ object.

Kinks on a straight step may have two opposite signs, "+" and "-" that are "looking" to the opposite directions along the step. Let us designate the number densities of these kinks, per unit length, by $\mathrm{n}_{+}$and $\mathrm{n}$. Density of the kink free sites is $\mathrm{n}_{0}$. Total density of the of contacts per unit step length is $n=1 / a$, so that

$$
n_{+}+n_{-}+n_{0}=n
$$

Then the free step energy per unit length is

$$
\gamma_{s t}=U-T S=(\varepsilon / 2)\left(n+n_{+}+n_{-}\right)-k T \ln n ! / n_{+} ! n_{-} ! n_{0} !
$$

Here $U$ and $S$ are the step energy and entropy, per unit length, and we ignore possibility of the kinks having the double, triple, etc depth. For simplicity, we again consider the step which, on average, keeps the $<100\rangle$ orientation on the (001) face, that is, $n_{+}=n_{-}=n_{ \pm}$. At equilibrium, kinks must be born and disappear keeping the step free energy at minimum: $\partial \gamma_{s t} / \partial_{n_{ \pm}}=0$. That minimization under the additional condition of eq.(25) provides the density of both the positive and negative kinks:

$$
a n_{ \pm}=\frac{2 \eta}{1+2 \eta}, \quad \eta \equiv e^{-\frac{\varepsilon}{2 k T}}
$$

and the average distance between the kinks

$$
\lambda_{k}=\frac{1}{n_{ \pm}}=a\left(1+\frac{1}{2} e^{\frac{\varepsilon}{2 k T}}\right)
$$

Combining eqs.(13),(20), and (28) we obtain the step propagation rate as a product of the driving force and kinetic coefficient, $\beta_{\mathrm{st}}$ :

$$
\mathrm{v}_{s t}=\beta_{s t} \omega\left(C-C_{e}\right), \quad \beta_{s t}=\left(a^{2} / \lambda_{k}\right) v^{-\frac{E-u_{M}}{k T}} .
$$


For the steps on prismatic face $\mathrm{KH}_{2} \mathrm{PO}_{4}(\mathrm{KDP})$, the activation barrier, $E-u_{\mathrm{M}}$, was measured to be $9.8 \mathrm{kcal} / \mathrm{mol}, \omega=9.68 .10^{-23} \mathrm{~cm}^{3} \sim 10^{-22} \mathrm{~cm}^{3}, C_{\mathrm{e}}=1.125 .1021 \mathrm{~cm}^{-3}, \beta_{\mathrm{st}}=7.8 .10^{-2} \mathrm{~cm} / \mathrm{s}$ at $\mathrm{T}=31^{\circ} \mathrm{C}(306 \mathrm{~K})$ [26]. With that activation energy, $v=6.10^{12} 1 / \mathrm{s}, \mathrm{a}=10^{-7} \mathrm{~cm}, \mathrm{a} / \lambda_{\mathrm{k}}=0.25$ (four lattice spacings between the kinks), kinetic coefficient $\beta_{\mathrm{st}}$ from eq. (30) is $\sim 2.10^{-2} \mathrm{~cm} / \mathrm{s}$, about 4 times less the experimental figure $7.8 .10^{-2} \mathrm{~cm} / \mathrm{s}$. Just to feel the numeric of the process, at $\omega C_{\mathrm{e}}=0.11$, and $\beta_{\mathrm{st}}=7.8 .10^{-2} \mathrm{~cm} / \mathrm{s}$ the exchange flux at the kink at equilibrium, eq.(22), is $w_{-} \sim 3.10^{4}$ units/s of which $\sim 1 \%$ is incorporated at the $1 \%$ supersturation. For the steps on the same prismatic face of the $\mathrm{NH}_{4} \mathrm{H}_{2} \mathrm{PO}_{4}$ (ADP), the step kinetic coefficient is $\sim 5$ times higher than for the KDP. The KDP vs ADP difference, as well as the inaccuracy in the eq.(30) should come from the simplicity of the assumptions on which this equation is based. In particular, we ignored difference in attachment/detachment rates of $\mathrm{PO}_{4}{ }^{3-}, \mathrm{HPO}_{4}{ }^{2-}, \mathrm{H}_{2} \mathrm{PO}_{4}{ }^{-}, \mathrm{K}^{+} . \mathrm{NH}_{3}{ }^{-}$, and several types of corresponding activated complexes, solution acidity controlling concentrations of the phosphate and hydrogen ions, difference in hydration heat between $\mathrm{K}^{+}(81 \mathrm{kcal} / \mathrm{mol})$ and $\mathrm{NH}_{4}{ }^{+}(78 \mathrm{kcal} / \mathrm{mol})$, difference in vibration frequencies $v$ of the ions present in the complexes, some, though probably small, difference in kink density. Evaluation of all these complexities is the matter of future.

The usually observed face growth rate is described by eq.(12). Together with eq.(30) it suggests that the kinetic coefficient for the face is just $p \beta_{s t}$ where $p$ characterises the step density (see Sec.2.5) Table2 summarizes the measured step and face kinetic coefficients for inorganic and protein crystals. Both coefficients for inorganic salts are typically 10-100 times lower than for proteins. The reason may be twofold. One, is larger entropic incorporation barrier for much larger protein ions bearing usually both positive and negative charges. Two, is larger potential energy barriers also stemming from the large size of protein molecules size.

Table 2.

\begin{tabular}{|c|c|c|c|}
\hline SUBSTANCE, FACE & $\beta_{\mathrm{st}}, \mathrm{cm} / \mathrm{s}$ & $p$ & $p \beta_{s t}, \mathrm{~cm} / \mathrm{s}$ \\
\hline $\begin{array}{l}\text { ADP, KDP, DKDP (100) } \\
\mathrm{NH}_{4} \mathrm{H}_{2} \mathrm{PO}_{4}, \mathrm{KH}_{2} \mathrm{PO}_{4}\end{array}$ & $(5-12) \times 10^{-2}$ & $3 \times 10^{-4}-8 \times 10^{-3}$ & $10^{-4}-10^{-3}$ \\
\hline ADP (101) & $0.4 \times 10^{-1}$ & $10^{-4}-5 \times 10^{-3}$ & $4 \times 10^{-5}-5 \times 10^{-3}$ \\
\hline $\mathrm{BaNO}_{3}(111)$ & $1.3 \times 10^{-2}$ & $(3-15) \times 10^{-4}$ & $4 \times 10^{-6}-2 \times 10^{-5}$ \\
\hline $\begin{array}{l}\mathrm{KAI}\left(\mathrm{SO}_{4}\right)_{2} \bullet 12 \mathrm{H}_{2} \mathrm{O}(111) \\
\text { alums }\end{array}$ & $8 \times 10^{-2}$ & $(0.4-3.5) \times 10^{-3}$ & $3 \times 10^{-5}-3 \times 10^{-4}$ \\
\hline $\mathrm{Y}_{3} \mathrm{Fe}_{5} \mathrm{O}_{12}(110),(211)$ & & $(0.3-3) \times 10^{-2}$ & $(0.4-1) \times 10^{-3}$ \\
\hline$(\mathrm{YSm})_{3}(\mathrm{FeGa})_{5} \mathrm{O}_{12(}(111)$ & $1.4 \times 10^{-2}$ & & $10^{-2}$ \\
\hline$(\mathrm{EuYb})_{3} \mathrm{Fe}_{5} \mathrm{O}_{12}(111)$ & & & $(0.1-3) \times 10^{-3}$ \\
\hline $\mathrm{CaCO}_{3}$ & $0.3-0.5$ & $3 \cdot 10^{-2}-10^{-3}$ & $10^{-2}-10^{-3}$ \\
\hline $\mathrm{Cdl}_{2}$ & $9 \times 10-3$ & & \\
\hline Brushite & $1.8 \times 10^{-2}$ & & \\
\hline GlyDKP & $3 \times 10-2$ & & \\
\hline & $3 \times 10-3$ & & \\
\hline a) lysozyme (101) 14,300 & $2-20 \times 10^{-4}$ & $(1.1-1.5) \times 10^{-2}$ & $3-30 \times 10^{-6}$ \\
\hline a) lysozyme (110) 14,300 & $2-3 \times 10^{-4}$ & $(1.0-1.5) \times 10^{-2}$ & $3-30 \times 10^{-6}$ \\
\hline b) canavalin $\quad 147,000$ & $9 \times 10^{-4}$ & $9 \times 10^{-3}$ & $9 \times 10^{-6}$ \\
\hline b) thaumatin & $2 \times 10^{-4}$ & 2D nucleation & \\
\hline c) catalase & $3.2 \times 10^{-5}$ & 2D nucleation & \\
\hline $1,600,000$ & $(4-8) \times 10^{-4}$ & 2D nucleation & \\
\hline e) ferritin & $6 \times 10^{-4}$ & 2D nucleation & \\
\hline
\end{tabular}


According to eq.(9), at the interface between crystal and aqueous solution the kink energy is $\varepsilon / 2 \approx \gamma a^{2}>k T$. This eq.(9) summarizes experimental measurements of the free surface energy from nucleation rate. Therefore the above estimate of the kink energy presumes low surface entropy determined by the kink density. This presumption is valid if the overall density of kinks on the interface is only a small portion of all molecular sites. This is certainly true for the smooth faces but may be less exact for the surface of a small nucleus. From eq.(9), the "dangling bond", kink and interface energy are the higher the lower the solubility and $\gamma a^{2} / k T>1$. The latter inequality suggests that the close packed lattice planes should be smooth. Indeed, the crystals in solutions are typically faceted, i.e. are below the roughening transition. Also, as it may be expected, the steps forming spirals around screw dislocations or islands from 2D nucleation are rounded on the faces of highly soluble salts, like KDP $\left(C_{e} \omega=0.11, C_{e}=1.1 .10^{21} \mathrm{~cm}^{-3}\right)$ and $\operatorname{ADP}\left(C_{e} \omega=0.22, C_{e}=2.10^{21} \mathrm{~cm}^{-3}\right)$ [26]. These steps are, however polygonized on the faces of sparingly soluble salts like biominerals. For example, hydroxyapatite, $\mathrm{Ca}_{5}\left(\mathrm{PO}_{4}\right)_{3} \mathrm{OH}$, has solubility product $\mathrm{K}=4.7 .10^{-59}$ (mol/liter) ${ }^{9}$ for all $5+3+1=9$ ions produced by one molecule, or $\mathrm{K}^{1 / 9}=3.310^{-7} \mathrm{~mol} / \mathrm{liter}=2.10^{14}$ ions $/ \mathrm{cm}^{3}$. Proteins have usually very moderate solubility of several mol\% but show both the severely kinked steps, like on the (111) face of ferritin (Fig.6) and the very straight steps with one kink per hundreds molecular spacing on lysozyme (Fig.7).

The kinetic coefficient eq.(30) was outlined assuming the equilibrium kink density. This density should be reached on step segments much longer than the distance between kinks and over the time essentially exceeding the time for creation or annihilation of a kink. However, the situation is different in the opposite case, $\varepsilon / 2 k T \gg 1$. That situation is expected and observed, for instance, on the faces of slightly soluble materials, eq.(9), Fig.7, on crystal - vapor face at low temperatures. Under those conditions, the interkink distance may reach $\mu \mathrm{m}$ scale and be comparable or even longer than the typical length scale of the interface micromorphology. For instance, it may exceed distance between impurities pinning the step or length of the first step segment of the polygonized spiral adjacent to the screw dislocation outcrop. The low kink density may also depend on supersaturation, as is discuss below.

At equilibrium, the average number of species in a crystal is preserved. Therefore some new kinks may be formed by two species detached from the "in the step" positions (leaving the $2 a$ long vacancy there), and then reattached at the neighboring kink free step segment. That process creates four kinks. Over the time, the kink generation by fluctuations is compensated by mutual annihilation of kinks of the opposite signs moving back and forth along the step. As supersaturation increases, more and more kinks are generated as "1D nuclei". Namely, imagine a first particle that is attached from solution on a straight kink free step segment. Unlike a particle at the kink, that "at the step" particle is bound by two rather by the three bonds. Therefore it has a larger chance to escape that a particle in the kink position. However, as soon as a second particle joins the first they both turn out to be in the kink position, so that two kinks are formed. A third particle has a chance to be attached to the pair and make a triplet before the pair loose one of the particles. This pair, triplet, etc. has the more chances to be saved in the crystal forever the larger the supersaturation and the longer the segment of the new row is. Evidently, at equilibrium, none of the attached particles will stay forever. 
Up to what supersaturation the equilibrium approximation for the kink density, eqs.(27),(28) is valid? Each kink propagate along the step at the rate $\mathrm{v}_{k}=a\left(w_{+}-w_{-}\right)$and simultaneously fluctuates with the diffusivity $D_{k}=a^{2}\left(w_{+}+w_{-}\right) / 2$. For mutual annihilation between the nearest positive and negative kinks they should cover the average interkink distance $\lambda_{\mathrm{k}}$. The time required for the annihilation of two kinks progressing at the relative rate $2 \mathrm{v}_{k}$ is $\lambda_{k} / 2 a\left(w_{+}-w_{-}\right)$. This time is long near equilibrium and quickly decreases as supersaturation rises. Annihilation may also occur due to diffusion and takes average time $\lambda_{k}{ }^{2} / 8 D_{k}=\lambda_{k}{ }^{2} / 4 a^{2}\left(w_{+}+w_{-}\right)$weakly dependent on supersaturation. At the steady state, the kink generation and annihilation rates are equal to one another while the balance should be controlled by the fastest annihilation path. Therefore the kinetic factors, including the 1D kink nucleation, rather than equilibrium fluctuations, should control the kink density and step rate if

$$
\left(C-C_{e}\right) / C_{e}>\sim 4 a / \lambda_{k}
$$

For orthorhombic lisozyme, at the interkink distance $\lambda_{\mathrm{k}}=75 a$, the kinetics should prevail at supersaturations $>\sim 5 \%$.

The rate of the "1D nucleation" on an infinite kink free step is $[27,28]$ :

$$
J(\text { nuclei } / \text { cm.s })=\frac{2\left(w_{+}-w_{-}\right) w_{+a}}{a\left(w_{-a}+2\left(w_{+}-w_{-}\right)\right)}
$$

where $w_{+_{a}}$, and $w_{-a}$ are the frequencies of attachment and detachment of the species at the step. In the same simple cubic lattice model, $w_{-a}=w_{-} \exp (\varepsilon / k T)>>w_{-}$. In the steady state, the kink density is constant, meaning balance between the 1D nucleation and mutual annihilation:

$2 n_{+} n_{-} \mathrm{v}_{k}=n_{ \pm}{ }^{2} \mathrm{v}_{k} / 2=J, \quad \mathrm{v}_{\mathrm{k}}=a\left(w_{+}-w_{-}\right)$.

Then the kinetic, rather the equilibrium, steady state kink density is:

$$
n_{ \pm}=\left(2 J / \mathrm{v}_{k}\right)^{1 / 2} \text {. }
$$

The kinetic density by eq.(34) may be shown to coincide with the equilibrium density eq. (27) at low supersaturation, but was not yet experimentally checked. The eqs.(33),(34) may be needed to discribe the weekly fluctuating straight steps at elevated supersaturations.

\subsection{Step Generation and Face Rate.}

Smooth crystal face cannot grow unless steps with the kinks are present on it. The steps may be generated by either 2D nuclei or a dislocation crossing the face and having a component $m h$ of its Burgers vector normal to the face. Here $h$ is the lattice spacing normal to the face and $m=1,2,3 \ldots$ or $1 / 2,1 / 3$, etc. Such a screw component of the Burgers vector generates the step(s) terminating at the point where the dislocation crosses the face. If $m>2,3, \ldots$ the dislocation outcrop typically is not a point. Rather, it occupies an area with a circumference $2 \mathrm{~L}$ of the order of a micrometer. As soon as supersaturation or 
supercooling is applied the step starts to propagate and forms a spiral around the immobile dislocation outcrop. If the kink density on the step is only slightly less than the reciprocal lattice spacing the step growth rate is isotropic within the face (Sec. 2.4). In this case, the spiral is rounded and forms a rounded pyramid. Steps with low kink density have anisotropic propagation rate over the face and the spiral is a polygonized pyramid. Such a pyramid is often called vicinal hillock. The slope of the pyramid, $p$, is typically small, $\sim 3.10^{-2}$ to $10^{-3}$. The slope determiners the power of the step generation and the face growth rate. If the spiral is rounded,

$$
p=m h /\left(19 \rho_{c}+2 L\right), \quad \rho_{c}=\omega \gamma_{s t} / h \Delta \mu, \quad \lambda=\left(9.5 \rho_{c}+2 L\right) / m
$$

where $\rho_{c}$ is the radius of the $2 \mathrm{D}$ nucleus and $\lambda$ is the distance between steps. It appearance in eq.(35) have nothing to do with the real nucleation event but comes from the Gibbs-Thomson law: the step at the point of dislocation outcrop is unable to move and thus must acquire the curvature of the 2D nucleus. The factor 19 may not be applicable to the polygonized pyramid since the Gibbs-Thomson law may not be applicable to the short step segments of which length is comparable with the interkink distance, Sec.2.4 [29]. In the case of the square spiral and pyramid,

$$
\lambda=\left(4 L_{c}+2 L\right) / m
$$

where Lc is the length of the squared critical nucleus. That is, the face rate is about twice as low than in case of the rounded vicinal hillock - at the same other parameters.

The step generation rate, that is the vicinal slope, $p$, increases about linearly with the low driving force, and reaches saturation at larger driving force, eq.(35),(36). The step propagation rate is the linear function of the driving force, eq.(30). Therefore the layer-by-layer growth rate of a crystal face increases as $(\Delta \mu / \mathrm{kT})^{2}$ at low supersaturation or undercooling and linearly at larger driving force.

Dislocation free crystalline faces grow by $2 \mathrm{D}$ nucleation. Their rate is practically zero at $\Delta \mu / \mathrm{kT}<\sim 1$ and increases exponentially at the higher driving force.

At sufficiently high supersaturation, more than $\sim 10 \%$ for KDP in solution or melt growth and probably > $\sim 100 \%$ in vapor growth or CVD, the 2D nucleation assures the step density exceeding that provided by screw dislocations. Therefore this is the the screw dislocation mechanism that is crucial for growth near the phase equilibrium while the 2D nucleation drives growth far away from equilibrium, mainly in MBE and CVD epitaxy, and from the vapor. The 2D step generation mode may be enhanced by defects lowering the $2 \mathrm{D}$ nucleation barrier.

Consideration in terms of activated complex at the kink, eq.(20), is valid also for layer-by-layer growth from a melt, for instance, for step propagation over the $\mathrm{Si}$ (111) facet of silicon, or for the layer-by-layer growth of Ga crystal, the rare metal having smooth interface in contact with its own melt. In one component systems, the potential barrier for crystallization might exist only if the interface is atomically smooth so that the layer-by-layer growth mode operates. This barrier is probably low and is of mainly entropic (ordering) nature, with some possible contribution from viscosity. In case of the rough interface, completely different mechanism discussed below in Sec.3 seems to operate. Kinetic coefficient for the step propagation at the crystal - melt interface is difficult to evaluate since it is hard to measure 
undercooling at the growing face. However, indirect estimates for the step kinetic coefficient on the $\mathrm{Si}(111)$ face growing from the melt is $\beta_{\mathrm{T}} \sim 50 \mathrm{~cm} / \mathrm{s} . \mathrm{K}$ [30], close to the coefficient roughly estimated to exceed $\sim 100 \mathrm{~cm} / \mathrm{s}$.K for metals. The measurement difficulties come, as usual, from the difficulty to evalueate the driving force immediately at the growing face or the step.

\section{NORMAL GROWTH MODE}

\subsection{Roughening Transition And Kinetic Roughening.}

The minimal free step energy, eq.(26), at the equilibrium kink density, eq.(27) reads:

$$
\gamma_{s t}=(k T / a) \ln \eta(1+2 \eta)
$$

As the kink energy to the thermal energy ratio, $\varepsilon / 2 \mathrm{kT}$, and thus its exponent $\eta$ decreases, the step free energy decreases. That means the meandering becomes easier, and the kink density rises.

Correspondingly, at the same absolute driving force, the steps grow faster. The free surface energy remains positive until $\eta=1 / 2$, or $\varepsilon / 2 \mathrm{kT}=\ln 2=0.69$. At the lower $\varepsilon / 2 \mathrm{kT}$ the step free energy, eq.(37) vanishes meaning transition from the smooth to the rough interface and, correspondingly, from the layerby-layer growth to the faster and transport controlled normal growth. Since the step free energy becomes small near the roughening transition the work to create a 2D nucleus on a smooth terrace and the nucleus radius also becomes small. These quantities vanish proportional to $\gamma_{s t}{ }^{2}$ and to $\gamma_{s t}$, respectively. As a result, the nucleus diameter becomes comparable with the molecular size. The density of nuclei becomes comparable with the density of molecular sites in the melt growth or the density of adsorbed species in the vapor or solution growth - even below the roughening transitions. In other words, the interface becomes rough for the kinetic reasons. This phenomenon is called kinetic roughening. The larger is supersaturation or undercooling the further below the thermodynamic roughening transition the kinetic roughening may occur. Onset of kinetic roughening enables morphological instability and growth of dendrites, difference in trapping of impurities and vacancies all over the growing interface and ultimately transition to the diffusion limited aggregation mode of deposition.

Crystallographic anisotropy of the roughening transition follows from more rigorous analysis [5] and is determined by the relationship between the roughening temperature $T_{R}$ and the distance $d$ between crystallographic planes parallel to the interface under consideration:

$$
k T_{R}=2 \bar{\gamma} d^{2} / \pi
$$

Here the surface stiffness $\bar{\gamma}=\left(\gamma+\partial^{2} \gamma / \partial \varphi^{2}\right)$ is taken at the orientation of the interface under consideration. It is also assumed that the stiffness with respect to the two major angles $\varphi$ characterizing deviations from this orientation is the same. The less compact the lattice plane the larger its Miller indices, the smaller is the interplanar distance $d$ and thus the roughening temperature. This seems to be the reason why equilibrium shape of small metal particles or tips, even in vacuum, is mainly about the sphere slightly cut by planes corresponding to the closest packed orientations with simple Miller indices. The mainly rounded shape is immediately replaced by polygon as soon as the crystal start growing. This fact beautifully demonstrates that the crystal growth rate is much more anisotropic than the surface free 
energy. In other words, the growth shape is controlled by the growth rate anisotropy rather than by the anisotropy of the surface energy. These two anisotropies are both controlled by the crystal structure so that the major closest packed planes may show up in both equilibrium and growth forms. However, size of different faces may be completely different.

\subsection{Normal Growth Of A Diffuse Interface.}

The term "normal growth" presumes that, opposite to the layer-by-layer growth, attachment of new spesies to the growing crystal occurs at any point of the interface rather than only at kinks at the steps. Therefore the normal growth does not need 2D nucleation of new layers and its dependence on the driving force is linear. The rough interface may be viewed as the lowest right image in the Fig.2. That model presumes that each atom is either "solid" or "liquid", that is, the crystal - medium interface is precisely localized down to atomic scale. In this case the rough interface is understood as the one where the fraction $\zeta=$ (number of "solid")/(number of "solid" + number of "liquid") atoms in each lattice plane parallel to the interface changes continuously from 1 in crystal to 0 in the liquid phase. The transition occurs within the layer of several, 5 - 10 lattice spacing thick. However, an isolated or nearly isolated atom cannot be solid or liquid. Therefore the transition from the crystalline order at $\zeta=1$ to the liquid disorder at $\zeta=0$ should be continuous on the atomic or molecular level, as shown in the upper portion of the Fig.8. In other words, on the way from the crystal to the liquid, each atom or molecule should become more and more delocalized via increasing the amplitude of its thermal vibrations [31,32]. That view of the really diffuse interface is consistent with molecular dynamic simulation [33, 34].

Within this picture, growth of the diffuse layer may be seen as simultaneous gradual formation of each plane parallel to the interface. The essential consequence of the delocalized interface concept is lack of activation barrier for the melt growth at the interface - for at least elements and sufficiently symmetric organic molecules. Indeed, in this case, the activated complex may be viewed as the whole transition layer built of numerous molecules thus smearing out the unique reaction path and the potential barrier.

Within the delocalized interface concept, growth rate of the interface is a product of the thermal rate of a free atom, the dimensionless driving force for crystallization and a constant:

$$
V=\text { const }\left(\frac{k T}{m}\right)^{1 / 2} \frac{\Delta \mu}{k T}=\text { const } \frac{\Delta_{S}}{k T}\left(\frac{k T}{m}\right)^{1 / 2} \Delta T
$$

The constant includes the sum of reciprocal lattice vectors of the growing crystal and the correlation length of particles in the liquid. The vectors present various lattice planes, which characterize the density waves of which superposition the crystal is to be built. Projections of these vectors to the normal to the growing interface determines the slight, $<10 \%$ crystallographic anisotropy of the growth rate. The correlation length represents the liquid structure and typical times of the order of $a /(k T / m)^{1 / 2}$. For $\mathrm{Pb}$, the latter time is $\sim 2.10^{-12}$ s. Some theoretical numerical estimates are seen in Fig.8 and may be found in $[31,32,33]$. The kinetic coefficient for lead was experimentally measured in [34] to be close to the predicted value, Fig. 8 . 


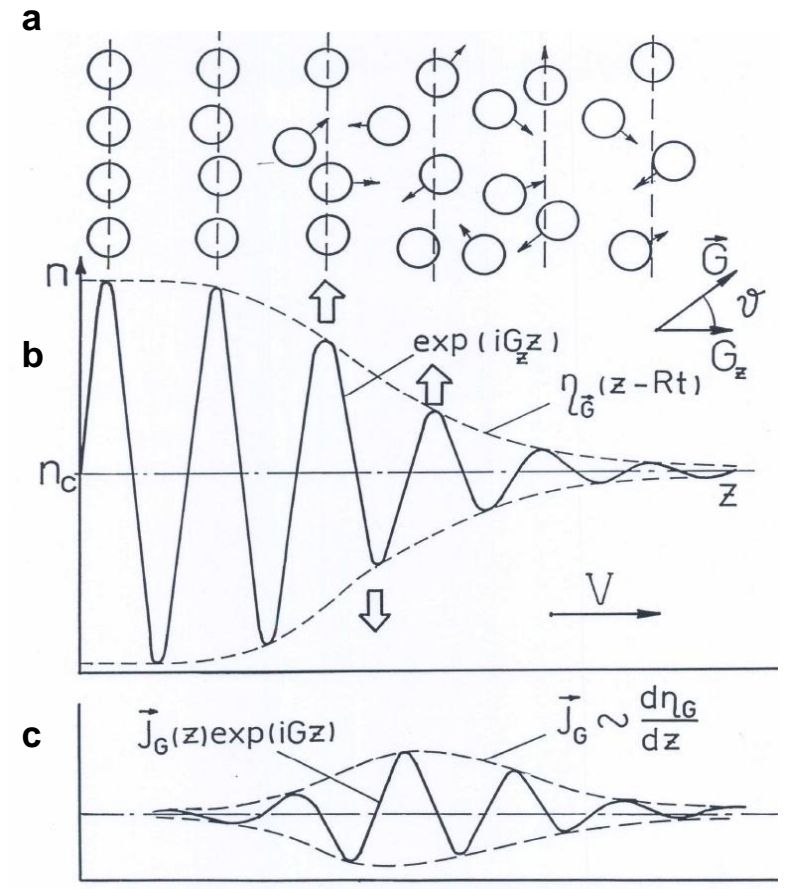

\author{
Several atomic planes \\ are being packed \\ simultaneously \\ Mikheev, Chernov, JCG 112(1991)591 \\ Normal growth rate: \\ $\mathrm{V}=\mathrm{b} \Delta \mathrm{T}=\mathrm{A}(\mathrm{kT} / \mathrm{m})^{1 / 2}(\Delta \mathrm{T} / \mathrm{T})$, \\ $\mathrm{A} \approx 1 ; \quad \mathrm{b}=\mathrm{A}(\mathrm{k} / \mathrm{mT})^{1 / 2}$ \\ Lead: \\ $\mathrm{m}=207 \cdot 1 \cdot 67 \cdot 10^{-24} \mathrm{~g}$, \\ $\mathrm{T}=600 \mathrm{~K}$, \\ $(\mathrm{kT} / \mathrm{m})^{1 / 2}=155 \mathrm{~m} / \mathrm{s}$ \\ $\mathrm{b}=26 \mathrm{~cm} / \mathrm{s} . \mathrm{K}$ \\ Experiment: \\ $\mathrm{b}=28 \pm 8 \mathrm{~cm} / \mathrm{s} . \mathrm{K}$ \\ Rodway, Hunt JCG 112(1991)554
}

Fig.8. Growth of a crystal from the melt within the diffuse layer intermediate between the crystal on the left and liquid on the right. The melt consists of simple particles symbolized by moving spheres in $\mathbf{a}$. Atomic planes shown in a correspond to the density waves $\mathrm{n}(\mathrm{z})$ shown in $\mathbf{b}$. Density waves $\mathrm{n}(\mathrm{z})$ are normal to the $\mathrm{z}$-axis in $\mathbf{b}$ and are presenting positions of the sphere centers subject to thermal vibrations. The wave period corresponds to the wave vector $\mathbf{G}_{z}$ of the reciprocal lattice of the crystal. The growth occurs via simultaneous ordering of the atoms into several crystal lattice planes $\mathbf{G}_{z}$. The growth front is normal to the z-axis and moving to the right at the rate $\mathrm{V}$. Atoms are not only being concentrated into the $\mathbf{G}_{z}$ planes normal to $\mathrm{z}$ to form this density wave. Simultaneously, ordering is going on within each plane so that the density waves with the wave vectors $\mathbf{G}$ different from $\mathbf{G}_{\mathrm{z}}$ are being built. As the growth precedes the amplitude of each wave rises from zero in the bulk liquid to the maximum within the crystal lattice. The panel $\mathbf{c}$ presents the ordering flux $\mathbf{j}_{\mathbf{G}}$ into the $\mathbf{G}_{\mathbf{z}}$ planes. The flux is proportional to the gradient of the order parameter $\eta_{\mathbf{G}}$ corresponding to the density wave normal to $\mathbf{G}$.

\title{
5. CONCLUSION
}

Current understanding of interface between crystal and surrounding vapor, melt or solution allows to estimate the interface energy, free energy of steps on smooth interface and to distinguish layer-by-layer from normal crystal growth mechanisms. The activated complex approach is instrumental in evaluation of step and face kinetic coefficients of crystallization within an order of magnitude. The diffuse interface concept provides more realistic view on the rough crystal - melt interface, predicts rather accurate growth kinetic coefficient and predicts negligible attachment/detachment barrier for simple liquids. This prediction is hardly applicable to a step of the singular interface.

\section{Acknowledgement}

This work performed under the auspices of the U.S. Department of Energy by Lawrence Livermore National Laboratory under Contract DE-AC52-07NA27344. 


\section{References}

1.Crystal Growth: An Introduction, Ed. P.Hartman, North Holland, Amsterdam, 1975. See also Proceedings of the 2-d to 13-th International Summer Schools on Crystal Growth.

2. D.Elwell, H.J.Scheel, Crystal Growth from High Temperature Solutions, Acad Press, London, 1975.

3. J.W.Mullin, Crystallization, Butterworth, London, 1972.

4. A.A.Chernov, E.I.Givargizov, K.S.Bagdasarov, V.A.Kuznetsov, L.N.Demianets, A.N.Lobachev, Modern Crystallography III. Crystal Growth, Springer Ser. Solid-State Sci, vol.36, Springer, Berlin, 1984.

5. A.Pimpinelli, J. Villain, Physics of Crystal Growth, Cambridge University Press, 1998.

6. K.A.Jackson, Kinetic Processes, In crystal growth, diffusion, and phase transitions in materials, Wiley-VCH, 2004.

7. Handbook of Crystal Growth, Ed D.T.J.Hurle, vols 1a,b, 2a,b, 3a,b North Holland, 1993-1994

8. W.K.Burton, N.Cabrera, F.C.Frank, Philos.Trans.Roy.Soc. A243,299(1951).

9. H.J.Leamy. G.H.Gilmer, K.A.Jackson, in: Surface of materials, vol.1, Ed J.M.Blackeley. Academic, NY,1975,p.121

10. M.C.Payne, N.Roberts, R.J.Neeeds, M.Needels, J.D.Joannopulos. Surf. Sci. 211/212(1989)1.

11. R.Roberts, R.J.Needs. J.Phys. Cond. Matt.1(1989)3139

12. M.A.van Hove, W.H.Weinberg, C.M.Chen, Low Energy Electron Diffraction, Springer, Berlin, 1986

13. L.E.Murr, Interfacial Phenomenain Metals and Alloys, Addison Wesley Pub Co, London, 1975.

14. A.A.Chernov, Contemporary Physics, 30, 4, 251-276(1989).

15. S.Toshev, in Crystal growth: An Intoduction, Ed P.Hartman, North Holland, 1973, 1-416. 16.

A.R.Miedema, Zs Metallkunde 69, 287(1978).

17. J.W.M.Frenken, Structure, dynamics, and melting of metal surfaces. Thesis, FOM, Amsterdam, 1986.

18. A.A.Chernov, V.A.Yakovlev, Sov Phys Doklady 30(11))1985)964; Langmuir 3(1987)635.

19. J.F.van der Veen, R.Pluis, A.W.Denier van der Gon, in: Chemistry and Physics of Solid Surfaces, vol VII, Ed R.Vanselow and R.F.Hove (Springer, Berlin, Heidelberg, 1988) p.455

20. O.Sohnel, J.Cryst Growth, 57,101(1982).

21. F.Rosenberger. Fundamentals of Crystal Growth, Springer Series Solid State Sc., vol.5,1979.

22. A.Yeckel, J.J.Derby, in: Bulk Crystal Growth of electronic, optical, and optoelectronic materials, Ed. P.Capper, Wiley, 2005.

23. I. Gutzow, J.Schmelzer, The Vitreous State, Springer, Berlin, Heidelberg, 1995.

24. S.-T. Yao, D.N. Petsev, B.R. Thomas, P.G. Vekilov, J. Mol. Biol. 303 (2000) 667

25. A.A.Chernov, L.N.Rashkovich. P.G.Vekilov, J Cryst. Growth, 275,1-18(2005)

26. L.N.Raskovich. KDP - Family Crystals. Adam Hilger, Bristol,1991. L.N.Raskovich. KDP - Family Crystals. Adam Hilger, Bristol,1991

27. V.V.Voronkov, Sov. Phys. Crystallography, 15,8(1973).

28. A.A.Chernov, J.Mater. Science: Materials for Electronics 12,437-449(2001).

29. A.A.Chernov, J.J.DeYoreo, L.N.Rashkovich, P.G.Vekilov, MRS Bulletin, 29,927-934(2004).

30. V.V.Voronkov, in Crystals: growth, properties, applications, vol. 9, Eds A.A.Chernov, H.MullerKrumbhaar, Springer,75-114(1983).

31. L.V.Mikheev, A.A.Chernov, J.Crystal Growth 112,591-596(1991).

32. A.A.Chernov, J.Crystal Growth, 264,499-518(2004).

33. A.Bonnisent, in Crystals:growth, properties, applications, vol.9, Eds A.A.Chernov, H.Muller- 
Krumbhaar, Springer, (1983)1-21(1983).

33. D.Y.Sun, M.Asta, J.J.Hoyt, Phys. Rev B69,024108(2004).

34. G.H.Roadway, J.D.Hunt, J.Crystal Growth,112,554(1991).

35. J.Christoffersen, E.Rostrup, M.R.Christoffersen, J.Cryst. Growth, 113,599-605(1991). 